\title{
Orthopaedic Modular Implants Based on Shape Memory Alloys
}

\author{
Daniela Tarnita ${ }^{1}$, Danut Tarnita ${ }^{2}$ and Dumitru Bolcu ${ }^{1}$ \\ ${ }^{1}$ University of Craiova, \\ ${ }^{2}$ University of Medicine and Pharmacy, Craiova, \\ Romania
}

\section{Introduction}

Intelligent materials are those materials whose physical characteristics can be modified not only through the charging factors of a certain test, but also through diverse mechanisms involving a series of additional parameters like luminous radiation, temperature, magnetic or electric fields etc. The use of intelligent materials in medical sciences offers to the economic medium the safest way to launch effective, highly-feasible and especially biocompatible products on the internal and international markets. The most important alloy used in biomedical applications is Ni-Ti, Nitinol (Nickel Titanium Naval Ordinance Laboratory), an alloy of an almost equal mixture of nickel and titanium, which is able to fulfil functional requirements related not only to their mechanical reliability but also to its chemical reliability and its biological reliability. Superelastic Nitinol alloys are becoming integral to the design of a variety of new medical products. The very big elasticity of these alloys is the most important advantage afforded by this material, but by no means the only or most important one. To highlight the value of superelastic Nitinol to the medical industry, we can present other properties: biocompatibility, kink resistance, constancy of stress, physiological compatibility, shape-memory deployment, dynamic interference, fatigue resistance hysteresis, and MRI compatibility (Duerig et al., 1999; Friend \& Morgan, 1999; Mantovani, 2000; Pelton et al., 2000; Ryhanen et al., 1999). These properties were used for manufacturing medical products including stents, filters, retrieval baskets, and surgical tools.

There are many metals exhibit superelastic effects, but only Nitinol based alloys is biologically and chemically compatible with the human body (Kapanen et al., 2002; Raghubir et al., 2007; Shabalovskaya, 1995; Yeung et al., 2007). In vivo testing and experience indicates that Nitinol is highly biocompatible, more so than stainless steel. The extraordinary compliance of Nitinol clearly makes it the metal that is most similar mechanically to biological materials. This improved physiological similarity promotes bony ingrowths and proper healing by sharing loads with the surrounding tissue, and has led to applications such as hip implants, bone spacers, bone staples, and skull plates. NiTi applications in orthopaedics currently include internal fixation by the use of fixatives, compression bone stables used in osteotomy and fracture fixation, rods for the correction of scoliosis (Yang et al., 1987), shape memory expansion staples used in cervical surgery (Sanders et al., 1993), staples in small bone surgery (Mei et al., 1997), and fixation systems for suturing tissue in minimal invasive surgery (Musialek et al., 1998). Several types of 
shape memory orthopaedic staples and plates for recovery of bones are used to accelerate the healing process of bone fractures, exploiting the shape memory effect.

\section{AO classification of human bones fractures}

In the AO classification system (Muller et al., 2006), for each long bone or bone group a number from 1 to 9 is assigned (Fig.1). The human long bones are: humerus-1; radius/ulna2; femur-3 and they are divided in three segments, designated by a number 1, 2 or 3; tibia/fibula-4 are divided in four segments, designated by 1, 2, 3, 4. A fracture is classified morphologically into three types in accordance with all the segments of the bone. The type of fracture is indicated by one of the following letters: A, B, C. Each type is divided into three groups: 1, 2 or 3 and each group is also divided into three subgroups, designated by: $.1, .2$, and .3. There are nine groups (A1, A2, A3 - B1, B2, B3 - C1, C2, C3) for each segment of the bone. The nine groups are organized according to severity criteria, as a function of the morphological complexity, the difficulty of the treatment and the prognosis, the gravity increasing from A1 (the most simple fracture) to $\mathrm{C} 3$ (the most complex and grave fracture). For exemple, the groups and the subgroups of tibial diaphyseal fractures are (Figure 3): 42. A1. Simple spiroid fracture; 42. A2. Simple oblique fracture $\left(>30^{\circ}\right) ; 42$. A3. Simple transversal fracture $\left(<30^{\circ}\right)$

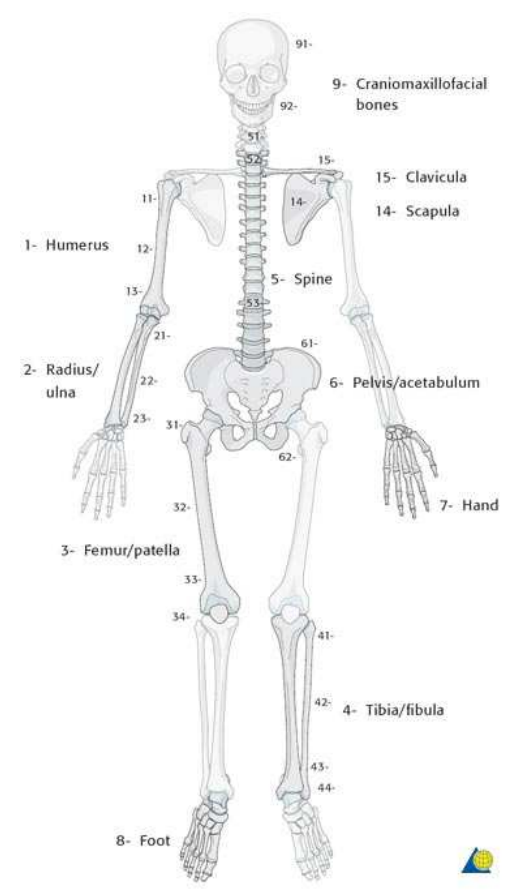

Fig. 1. AO system for numbering the anatomical location of a fracture in three bone segments (proximal=1, diaphyseal $=2$, distal $=3$ ); where the assigned numbers for human bones are: humerus-1; radius/ulna-2; femur-3; tibia/fibula-4; spine-5, pelvis-6, fand-7, foot-8, craniomaxillofacial-9 [Muller et al., 2006 ] 
The anatomical location of the fractures for long bones is presented in Fig. 2 and the AO classification of the type A tibial diaphyseal fractures is presented in Fig.3 (www.aofoundation.org).
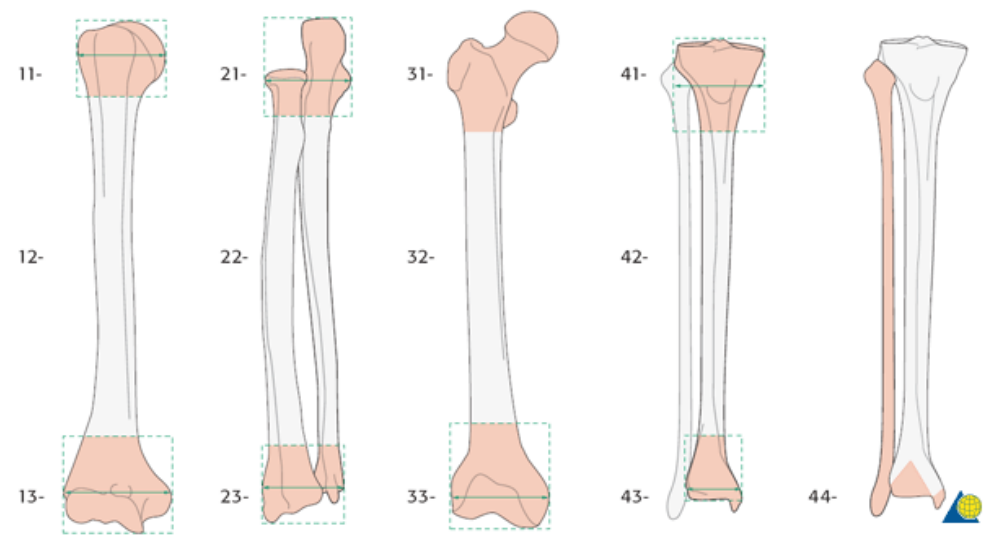

Fig. 2. Anatomical location of a fracture is designated by two numbers: one for the bone and one for its segment: 1.1. proximal humerus; 1.2. diaphyseal humeru; 1.3. distal humerus; ulna and radius are regarded as one bone: 2.1. proximal radius+ulna; 2.2. diaphyseal radius +ulna, 2.3. distal radius +ulna); 1.1. proximal femur; 1.2. diaphyseal femur; 1.3. distal femur; 4.1. proximal tibia+fibula; 4.2. diaphyseal tibia+fibula, 4.3. distal tibia+fibula;

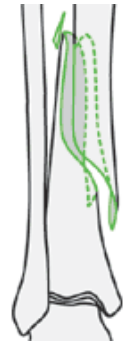

42-A1.1;

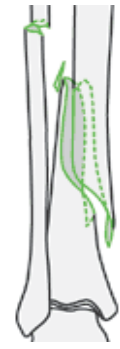

42-A1.2;

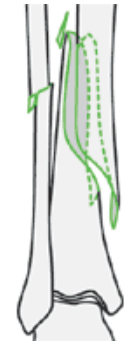

42-A1.3;

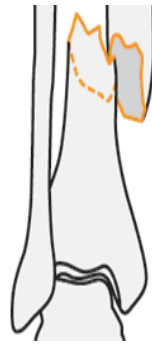

42-A2.1;

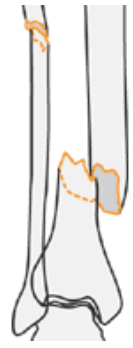

42-A2.2;

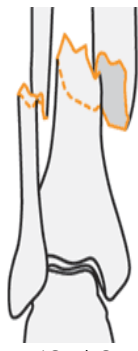

42-A2.;

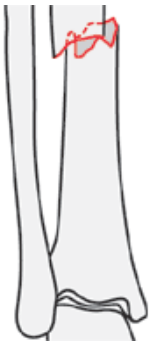

42-A3.1;

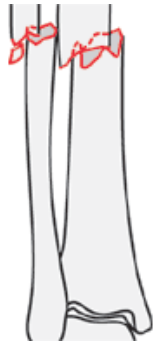

42-A3.3

Fig.3. AO classification of the type A tibial diaphyseal fractures: A1- Simple spiroid fracture; A2-Simple oblique fracture; A3-Simple transversal fracture; 42-A1.1 Fibula intact; 42-A1.2; Fibula fractured at other level; 42-A1.3 Fibula fractured at the same level; 42-A2.1 Fibula intact; 42-A2.2 Fibula fractured at other level; 42-A2.3 Fibula fractured at the same level; 42A3.1 Fibula intact; 42-A3.3 Fibula fractured at the same level

A comparative analysis of 2700 diaphyseal bone fractures shows that each type of fracture occurs with similar incidence in the case of the four bones: A type: $61 \%$ for humerus, $65 \%$ for radius/ulna, $53 \%$ for femur and $45 \%$ for tibia/fibula; B type: $32 \%$ for humerus, 29 for radius/ulna, $34 \%$ for femur and $46 \%$ for tibia/fibula; $C$ type: $7 \%$ humerus, $6 \%$ radius/ulna, $13 \%$ femur and $9 \%$ tibia/fibula. The fracture groups have a similar distribution for humerus, femur and tibia/fibula. For example, the spiroid fractures take $27 \%$ from the humeral fractures, 23\% from the femoral fractures and 25\% from tibial fractures (Muller et al., 2006). 


\section{Three dimensional virtual models for human bones}

The geometry and mechanical properties of the bone system vary naturally by individual which can create great difficulties in biomechanical research. The dimensions, form, mechanical properties, elastic constants, physical constants of the bone are different for different individuals. They depend on: age, sex, height, profession etc. The geometrical aspects of the bone systems modelling are dominated by the necessity of using some spatial models because most of the bone elements have complicated geometrical forms in space. The creation of the virtual 3D model of the human bones and the numerical simulations require:

a. Three-dimensional modelling of the bones -using parametrical CAD software, SolidWorks or Catia.

b. Mesh generation of each virtual model of human bones in finite elements (using tetrahedral 3D elements).

c. Establishment of the contour conditions

d. Introduction of the mechanical parameters for each material (cortical and spongy) of the bones

e. Finite element method analysis and simulation with dedicated software (ANSYS, VisualNastran)

To obtain the three-dimensional virtual models, bones from dead persons, kept in the Laboratory of Anatomy from the University of Medicine and Pharmacy, Craiova were used. To obtain the bone cross sections a PHILIPS AURA CT tomograph installed in the Emergency Hospital from Craiova was used. The obtained images were re-drawn in AutoCad over the real tomographies and the drawings were imported in SolidWorks (a parametrical CAD software), section by section, in parallel planes. Solidworks allows the creation of a solid by "unifying" the sections drawn in parallel planes (www.solidworks.com). The feature we used the Loft Shape and it defines the solid starting with the sections and using a Guide Curve defined automatically by the software. Finally, we obtained the spatial virtual models of the studied bones. The bones have been modelled, mainly, through a cylinder of compact bone tissue having a central canal called medullar cavity which penetrates the extremities where the model narrows due to some lamellar systems. The extremities of the bone are made from a thin layer of compact bone substance (cortical) at the exterior and a spongy material at the interior.

For the next step we used the ANSYS program to obtain the mesh structure with finite elements method of the spatial structure of bones (Tarnita et al., 2009). The threedimensional modelling of the bone structures has been succeeded by the introduction of a series of physiological and morphological parameters in order to allow a corresponding study of the bones at different stresses and deformations that characterise both normal situations and accidents.

The next step was to apply the material properties to the virtual models by introducing the elastic constants (elasticity modules and Poisson coefficient) by considering the nature of the bone material: a composite material made from cortical and spongy. The values for the longitudinal elasticity modules and Poisson coefficient have been chosen from the literature.The work hypotheses are:

a. Even though the material of the bone is an-isotropic and not homogeneous, in the modelling, the bone was considered homogeneous and isotropic, for a zone of solicitation that does not exceed certain limits.

b. The bone is made by two kinds of materials, compact and spongy, like a composite material. 
c. The average values considered for the longitudinal modulus of elasticity, E, are: 18.500 $\mathrm{N} / \mathrm{mm}^{2}$ for the compact bone, situated in the exterior zone of the bone and $2 \mathrm{~N} / \mathrm{mm}^{2}$ for the spongy bone, situated in the interior zone. The value of the Poisson coefficient was 0.3 .

d. The virtual central canal is realized in accordance with the obtained tomographies, so the complex spatial structure is ensured.

Ulna: The ulna is a long bone of the forearm, broader proximally, and narrower distally. Proximally, the ulna has a bony process, the olecranon process, a hook-like structure that fits into the olecranon fossa of the humerus. This prevents hyperextension and forms a hinge joint with the trochlea of the humerus (Gray, 2000). There is also a radial notch for the head of the radius, and the ulnar tuberosity to which muscles can attach. Distally (near the hand), there is a styloid process. The long, narrow medullary cavity is enclosed in a strong wall of compact tissue which is thickest along the interosseous border and dorsal surface. At the extremities the compact layer thins. The compact layer is continued onto the back of the olecranon as a plate of close spongy bone with lamellae parallel. The sections of ulna bone realized in SolidWorks are presented in Figure $4 \mathrm{~b}$. Finally, it is obtained the virtual model of the ulna bone (Figure 4c).

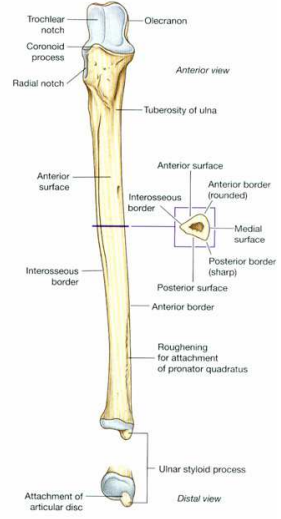

a)

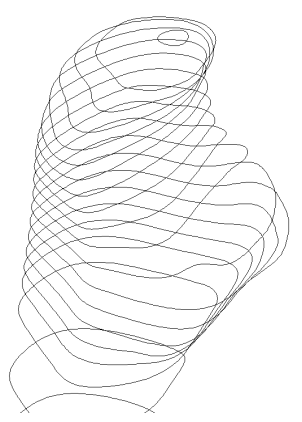

b)
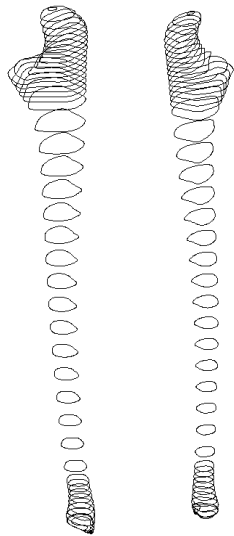

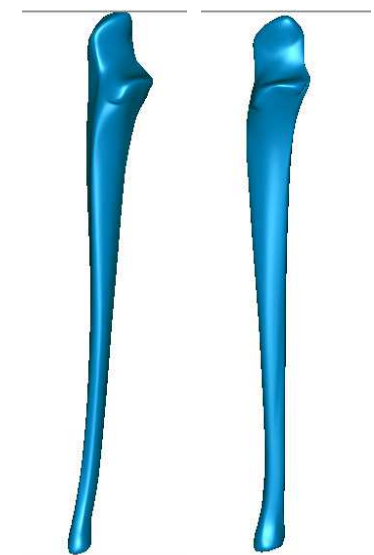

c)

Fig. 4. Ulna bone (a) (Gray,2000), sections of ulna bone (b), the virtual model of ulna bone (c)

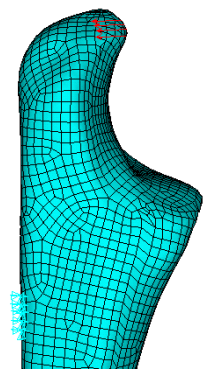

a)

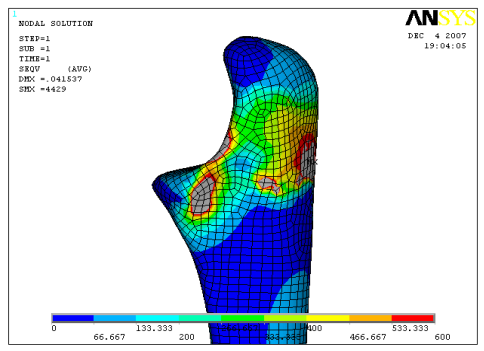

b)

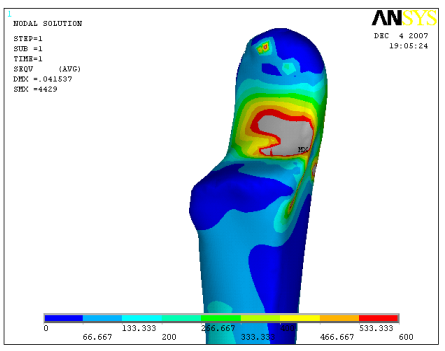

c)

Fig. 5. The loading forces (a) and the resulted stress maps (b and c) for the first case 
The mesh structure is presented in Fig. 5a. The bone is considered leaned on its metaphyseal area. The geometrical model was subjected to a solicitation equal to a force of $1000 \mathrm{~N}$, in two cases, like in Fig. 5a, and Fig.6a.

In Fig.5b and Fig.5c the stresses diagram for first loading case were obtained. In Fig.6 are presented the stress diagrams for the second loading case. We can see that the maximum values of stresses appear in the articular portion of the proximal ulna. They may involve the olecranon process or the coronoid process. This area is the most solicited part of the bone which is more likely to be involved in fractures.

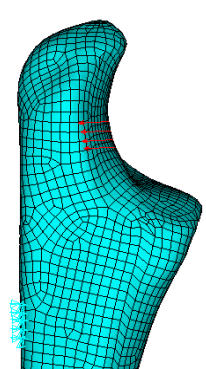

a)

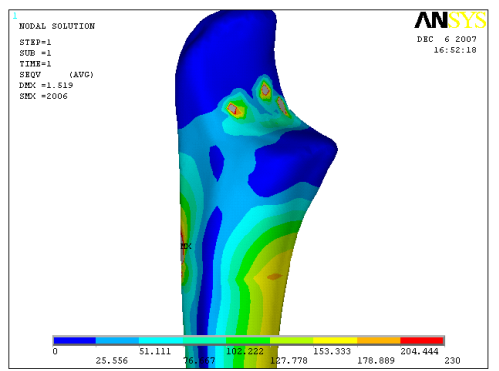

b)

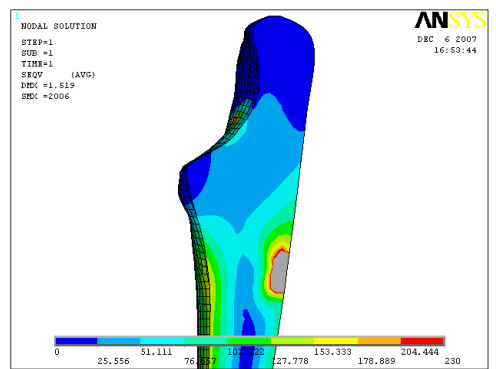

c)

Fig. 6. The loading forces (a) and the obtained stress maps (b and c) for the second loading case

Tibia. In the first case the tibia bone was subjected to a torsion couple on the top surface (Fig.7a.). The bone is supported on its inferior base. We obtained the resultant stress distribution for the torsion solicitation for the entire bone (Fig.7b). and for a section made in this bone (Fig.7c). In the second case the bone was supposed to the bending by a normal force equal with $180 \mathrm{~N}$ distributed on the middle of tibia bone. The bone is leaned in his both heads. We obtained the resultant stress distribution for the bending solicitation (Fig.8a.). In third case the bone was supposed to a compression force distributed on the both superiors condils (Fig.8b.). Finally, we obtained the resultant stress distribution for the compression solicitation (Fig.8c.).
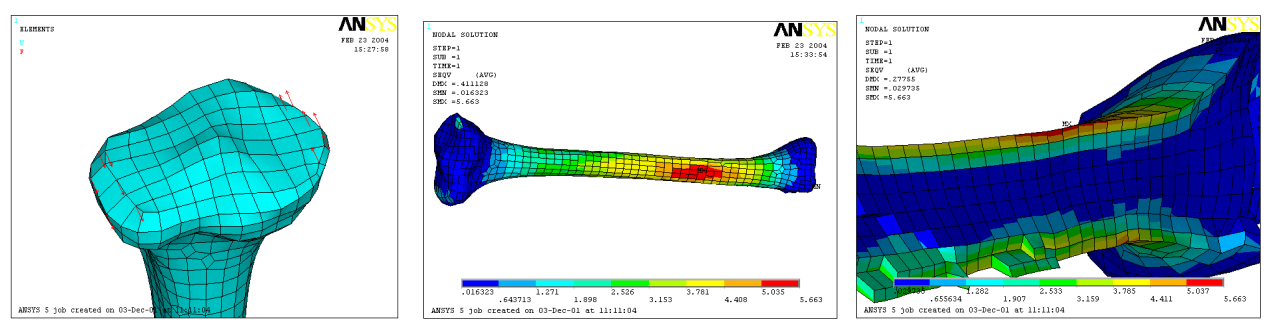

Fig. 7. The torsion solicitation (a) and the stress diagram obtained for the entire bone (b) and for a section of the bone (c) 


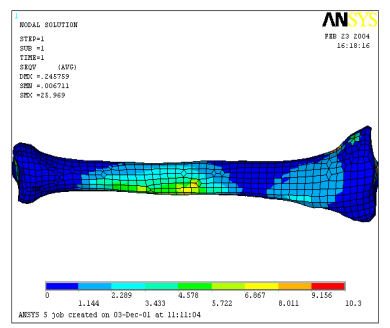

(a)

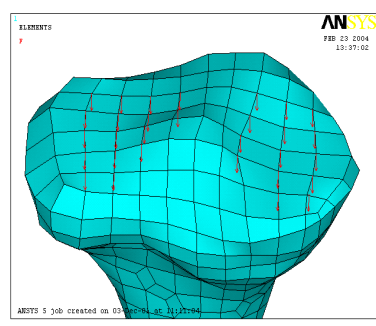

b)

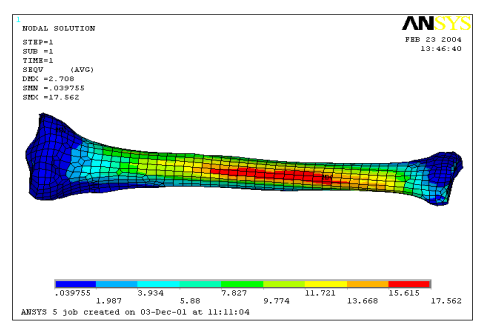

c)

Fig. 8. The stress map for the second case(a), the loading force in the third case (b) and the stress map obtained for the third case (c)

Metacarpi: The virtual model of the bone was subjected to:

- traction with a force evenly distributed on the head of the metacarpal bone surface, while the basis is the fixation surface. The stresses diagram can be observed in Figure 9a and the displacements diagram in Figure $9 \mathrm{~b}$.

- torsion with a moment applied at the metacarpal head, the basis of the bone being fixed. The tension diagram is given in Figure 9c and Figure 9d.

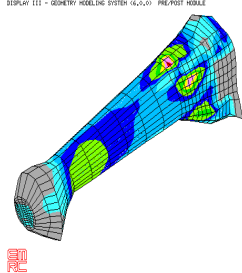

a)

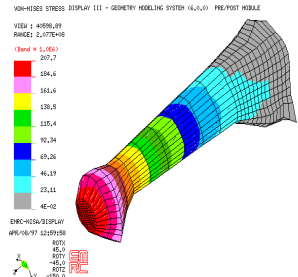

b)
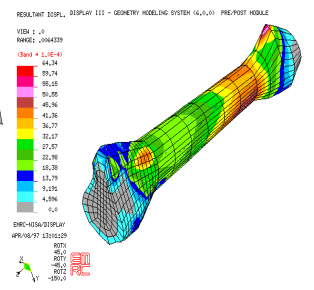

c)

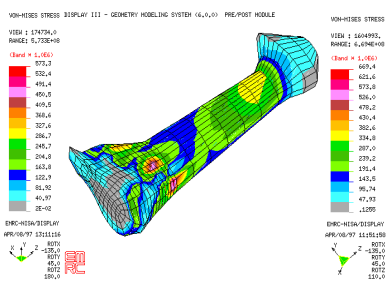

d)

Fig. 9. The stresses diagram (a), the displacements diagram (b) for traction and stresses diagram for torsion solicitation of the metacarpi bone (c and d)

Clavicle:Two loading cases have been taken into consideration:

1. compression with an evenly distributed force equal with $4 \mathrm{~N} / \mathrm{mm}^{2}$ applied on the extreme surface of the clavicle while the basis is the fixation surface (results in Figure 10a and Figure 10b).

2. flexion given by $400 \mathrm{~N}$ of force distributed on the lateral surface of the clavicle (results in Fig.10c and Fig.10d)

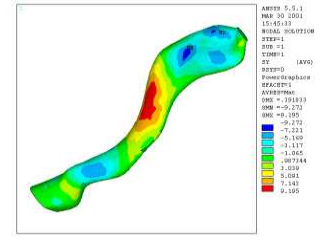

a)

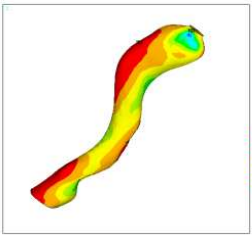

b)

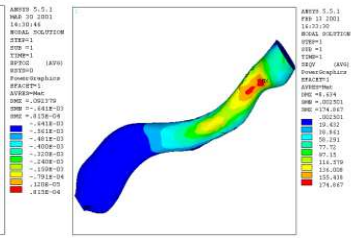

c)

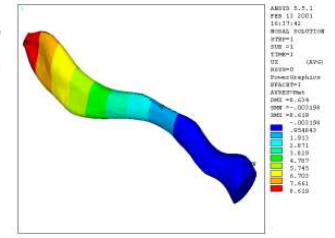

d)

Fig. 10. The normal tension map for clavicle (a), the linear deformations for the clavicle bone in the first case (b), and tension map (c) and deformation map (d) in the second case 
Phalanx: The virtual model of the phalanx has been tested to:

1. compression with a force evenly distributed on the superior surface of the phalanx head, while the bone is fixed at the basis (Fig 11 a- The stress diagram; Fig 11 b- The displacements diagram);

2. bending given by a force which acts at the middle of the bone, both extremities (proximal and distal) being fixed (Fig 11 c- The stress diagram; Fig $11 \mathrm{~d}$ - The displacements diagram);

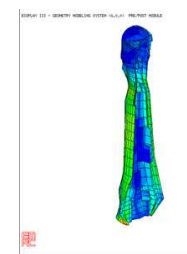

a.

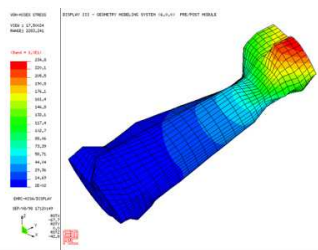

b.

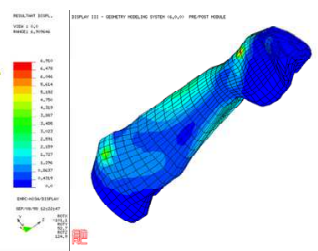

c.

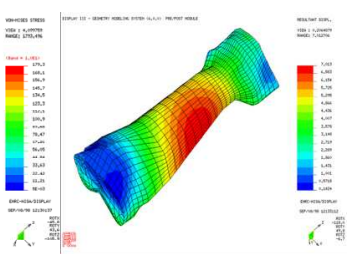

d.

Fig. 11. The results obtained by numerical simulations for the above considered loading cases for the phalanx bone

Radius: In Fig. 12.a. we present the mesh structure of the virtual model of the radius bone. In Fig.12.b, a longitudinal section through radius is presented. The virtual model was tested with a moment of $4.8 \mathrm{Nm}$ (torsion) applied on the surface of the radius head. The bone is fixed at the inferior side. The virtual stress is given by a set of forces placed at the extremities of the radius head in a plane normal to the longitudinal axis of the bone. The stress diagrams are shown in Fig.12.c, while the deformations diagrams in Fig.12.d.

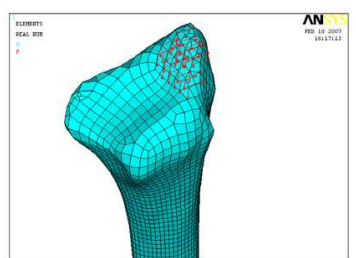

a.

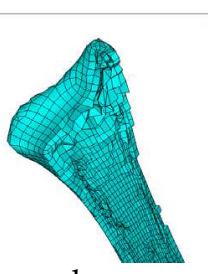

b.
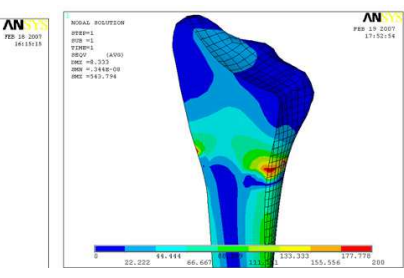

C.

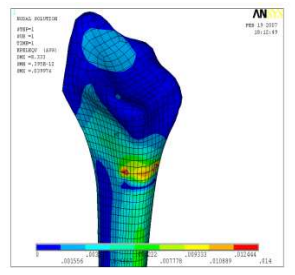

d.

Fig. 12. The virtual model of the radius (a), longitudinal section through radius (b) the stress map (c) and deformation map (d)

Analysis of stresses and deformations of the fracture areas is carried out with the Finite Element Method which allows: accuracy of the complex bony structure geometry, variation of designing parameters, and combination with other bone modelling algorithms leading to various types of analyses. The software allows the determination of the stress and displacements distribution in some particular cases which determine the breaking of the ulna. The correspondence between the clinical observations, virtual simulations and the results obtained from the experimental tests using the universal testing machine EDZ20 certify the accuracy and the fidelity of the spatial geometry, but also of the bone structure, of the mechanical properties of the bone. 


\section{Orthopaedic modular implants based on shape memory alloys}

Treatment of bone fractures depends on the stability of the fracture configuration. The fixation of the fracture must sometimes accomplish a reduction of the fragments and to spare the vasculature of these fragments as much as possible. It is well known that stable fixation and compression are the two main factors in obtaining union between two bony fragments. The choice of fracture treatment, orthopaedic or surgical and also the type of surgical treatment must take into account the type of fracture, the importance of displacements, the skin condition, patient age and also the patient's possibilities and his willingness to cooperate. A stable fracture can be adequately managed with cast immobilization. An unstable fracture may require surgical fixation. In many cases, the treatment of fractures involves surgical intervention to stabilize the fracture. The traditional method of open reduction and plate fixation requires wide exposure of the fracture site with stripping of the soft tissues which may devascularize the fracture fragments (Heim \& Pfeiffer, 1988). This may contribute to the necrosis caused by trauma and increase the risks of delayed healing and infection.

In selecting the optimum technique, the surgical complexity, mechanical performance, and biological response should be considered. Typically, a fractured or cut bone is treated using a fixation device, which strengthens the bone and keeps it aligned during the healing process. Mechanically, the fixation should provide sufficient stability. Biologically, the treatment should be minimally invasive, the implants well tolerated, and the resulting bone stresses optimal for fracture healing. Even if it's well known how important the integrity of soft tissues is during surgical fracture treatment, by plate-screw fixation, trauma surgeons have always had a tendency to reach maximum biomechanical stability, regardless of the impact on bone vasculature. The conflict between the anatomical needs to reduce the distance of the fracture and the desire to keep the vascularisation of all bone fragments is the reason for the appearance of minimal invasive surgical techniques. Techniques of minimally invasive surgery were developed to avoid wide exposures of the fracture site and minimize soft tissue damage (Farouk et al., 1999). They are described for some fractures in the lower extremity (Kregor at al., 2001). The implants of classical materials have the following disadvantages, clinically observed, in time, by the orthopaedic specialists:

1. Relatively large dimensions of implants due to respecting resistance conditions to materials' breaking norms, classical materials have break-resistance values much more inferior to alloys' values with shape memory;

2. The augmentation of the implants dimensions determines large incisions with large blood and soft tissues losses, and aggravating conditions of the bone, which lead to consolidation delays but also to increased infection risk;

3. The healing process becomes complicated due to lack of fracture components' compaction, fact which often leads to consolidation delays and even pseudo-arthrosis;

4. Multiple holes where implant-fixing screws are introduced represent tension concentration which leads to bones' fragility and appearance of new fracture focuses;

5. They are supplied with differently typo-sizes, separately for each type of bone, for each bone area for each fracture type and size separately

6. Using compaction devices designed to improve the rate of healing percentage when plate screwed fixation is used, cause scarring even longer and destruction of soft tissues greater than plate screwed fixation without compaction. 
7. Because of cortex sponging the boards need to be extracted (which means a second surgery). By extracting, there is a fracture risk of one of the holes for screws, causing peri-fracture tissue damage and peri-implant as big as or bigger than the implant operation. In order to eliminate or at least reduce inconveniences caused by the lack of compaction, plates with screws with compacting or self compacting have been created. These plates, however, fail to achieve a satisfactory compaction and, in addition, require larger incisions and with greater tissue damage, higher blood loss and increased exposure to infections and scarring are larger and more unsightly and compaction in this case is achieved with the bone fixation and not continuously, as with the model proposed by us.

8. All inconveniences, besides prolonging patient's suffering, increase the number of hospital days as well as the number of disability days at work, leading to high social costs.

\subsection{Orthopaedic modular plates based on shape memory alloys}

The classical bone plates with screws prevent bone compaction and do not allow application of axial forces caused by muscle tension in normal bones which leads to the delay of fracture focus consolidation or leads to a non-union (pathological neo-articulation). The classical metal plate used as an implant must be sufficiently large to achieve solid fixation of the fracture fragments. Current orthopaedic plates use titanium or special steel, materials which are subject to electrolytic action of the biological environment, without allowing a pseudo- elastic behaviour similar to bone structure. Because the lengths of the classical plates are big, the surgery for metallic implant mounting needs large incisions with great tissue damage, with great loss of blood, tissues with high exposure to the environment, which increases the risk of infections, with big risks in their propagation to the bone (bone infections are incurable) and obtain scarring. Internal tissues are exposed to foreign microbial increasing the danger of infecting the wound. The implant has a large contact surface with the biological environment which increases the risk of rejection by the body or occurrence of inflammatory phenomena. These requests affect the process of bone recovery leading to the appearance of a bony callus formed incorrectly, to structural goals or to geometrical deformations of fractured bone. Another disadvantage is related to plates' reduced adaptability to the specific particularities of each fracture case occurred in practice. The only degree of adaptability allowed to the current plate-type implants is provided by using additional holes which allow fixing screws depending on the size of fracture/fractures.

For modelling the optimum implant shape according to the type of fracture, it is taken into consideration the simulation with Finite Element Method of the various areas where the implants are to be placed. Studies continue with modelling various implant shapes and their experimentation in virtual environment in order to determine optimum shapes to provide perfect interweaving of fractured bony structures. The optimum shape has to take into consideration the implant insertion technique as well. The proposed implants have a modular design, with memory shape as elements of module coupling. The design of proposed modular implant involves a minimal invasive implantation, small dimensions, which can be coupled intra-operatory, in order to obtain modular plates of various lengths and configurations appropriate to the fracture. 
The modular structures for implants are used for the osteosynthesis of diaphyseal and metaphyseal fractures of long bones. These are based on the making of identical modulescompletely interchangeable, made of titanium or biocompatible stainless steel 316L, after which Nitinol elements are interconnected. The shape memory effect in the case of a staple is connected with a contraction of the fixative, enabling not only reduction or elimination of a gap between the bone fragments to be joined, but also appropriate compression. This system includes a multitude of identical linear modules which correspond to the diaphyseal area of the bone, as well as a multitude of nonlinear modules with different dimensions corresponding to the epiphyseal areas of the bone. These modules can be manufactured in shapes and dimensions compatible with the area of the bone undergoing surgical intervention. They have a particular type of shape which allows for an initial coupling by translation, the final coupling and fracture compacting being aided by memory shape staples. The shape and the dimension of the plates can be adjusted to fit any bone type and fracture location, allowing the surgeon to improve the alignment of the fractured bones and the distance between them.

Modular plates have the task of fixing and stabilizing the fracture centre and can be mounted on the bones via a well established procedure. The surgeon must: 1) select the appropriate module, 2) reduce the fracture fragments; 3 ) secure the plate modules onto the two fragments on either side of the fracture with screws and 4) compact the fracture by coupling the modules using memory shape elements. The modules are made from biocompatible materials with adequate mechanical properties (titanium and titanium alloys, cobalt, stainless steel, ceramic materials). The plate axis coincides with the bone axis. The length and/or width of the modules can be different from one application to another. Generally, the modules are linear, for using on the diaphyseal portion of long bones, but they can also be nonlinear for the bone heads, being configured and dimensioned in a nonlinear shape which best suites the epiphyseal bone areas. These nonlinear modules have a transversal portion of corresponding shape and dimensions and an axial portion to ensure the attachment with a linear module to the diaphyseal portion of the bone.

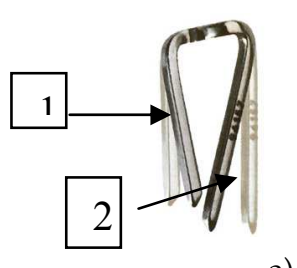

a)

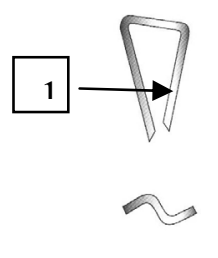

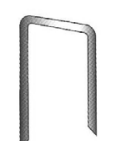

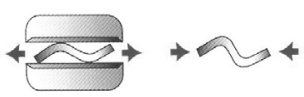

b)

Fig. 13. The schema process of staple shape transformation: a) the two positions of the staple: 1)-initial and 2)-final; b) the transition process from the austenitic stage 1) (high temperature) to the martensitic stage 2) (low temperature)

The U-shaped staple has two straight sides and a middle "active" section pre-deformed by tensile stress. The connection of the modules is made by inserting the staple pins in their open form (at low temperature, in the martensitic state) in the channels of the modules. After implantation, the staples return to their initial form under the influence of body heat, thus closing the space between bone fragments. The open structure is designed to stabilize and stiffen the montage and allows for a sliding motion along the longitudinal axis of the 
bone which coincides with the plate axis and allows for the compacting process for the two bone fragments. The schema process of staple shape memory transformation is presented in Figure 13 (www.groupe-lepine.com) Due to its pseudo-elastic property, a memory-alloy staple maintains a compressive effect ensuring a constant compressive force between the two modules and, thus, between the two bone fragments. This way, the staple forces a bone alignment very close to the normal anatomical alignment of the bone, which is highly conducive to cellular regeneration and healing. After the fracture is healed, the staple can be cooled, thus returning to its open form, allowing for an easy extraction. The modules may also be extracted easily by the surgeon.
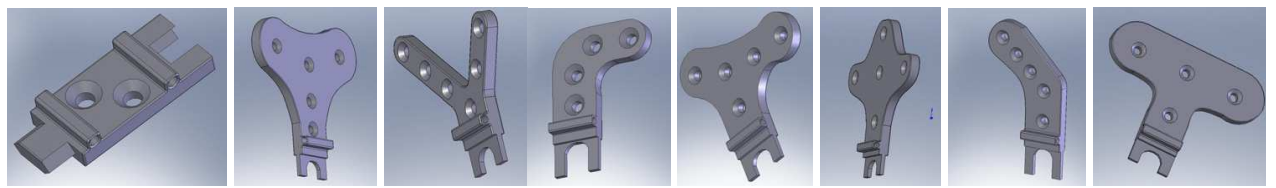

Fig. 14.Various diaphyseal and epiphyseal modules
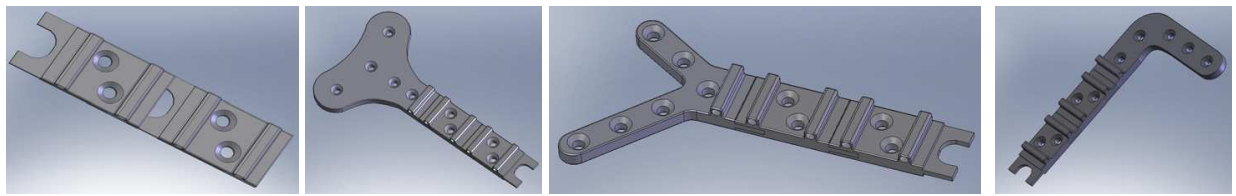

Fig. 15. Various types of modular plates for diaphyseal fractures (a) and for metaphyseal and epiphyseal fracturesa $(b, c, d)$

Using specialized software as VisualNastran [18-19], and the principle of the von Mises stress, the numerical simulations movies of the assembly fractured tibia-modular plate are obtained. In materials science and engineering the von Misses yield criterion (von Mises, 1913) can be formulated in the following way: a material is said to start yielding when its von Misses stress reaches a critical value known as the yield strength. The von Misses stress is used to predict yielding of materials under any loading condition from results of simple uniaxial tensile tests.

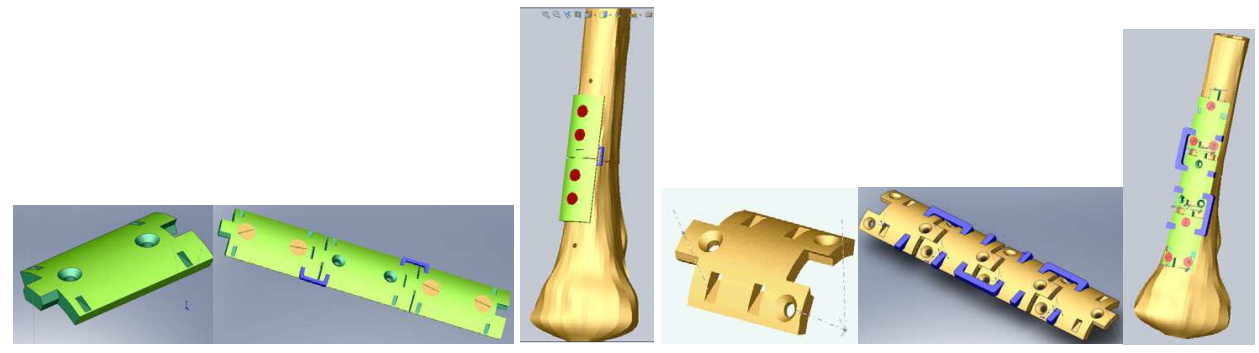

Fig. 16. Modules, plates and tibia-modular plate assembly for diaphyseal fractures

A compression force of $54 \mathrm{~N}$ was applied on the extremities of the staples which connects the modules. In Fig. 17 the stress maps and the displacements maps for two succesives moments of the implant assembly are presented 


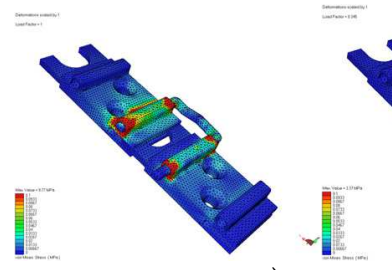

a)

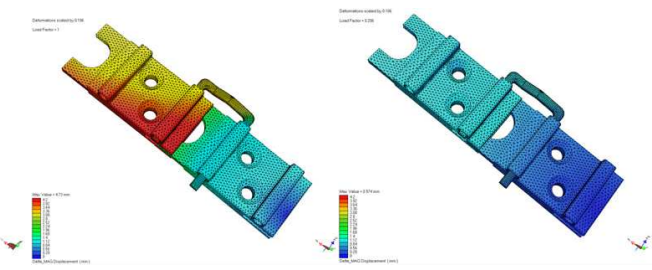

b)

Fig. 17. The stress (a) and displacements (b) maps for the implant assembly

In Figure 18 a)-c) are presented three different stages of the numerical simulation movie of the assembly tibia bone-virtual modular plate for each kind of diagram: von Mises stress diagram [Pa], displacements diagram $[\mathrm{mm}]$ and von Mises strain diagram $[\mathrm{mm} / \mathrm{mm}]$. In Fig.18 d) two stages of the von Mises stress diagram for the staple are presented. These diagrams show the variation of the values during the simulation of the staple shape transformation from the martensitic stage to the austenitic stage.

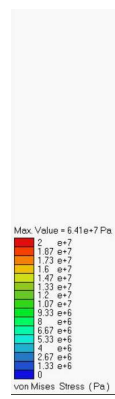

a)

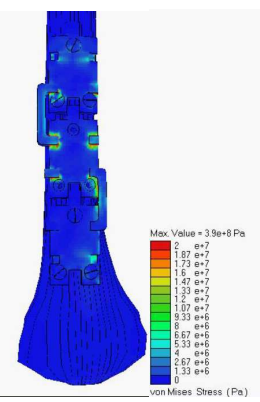

b)

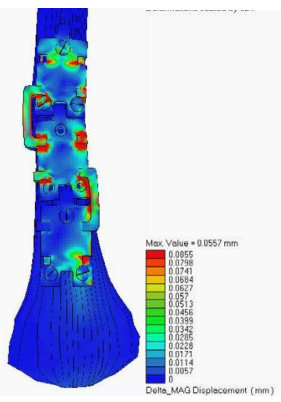

c)

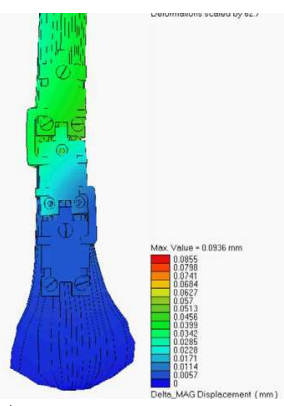

)

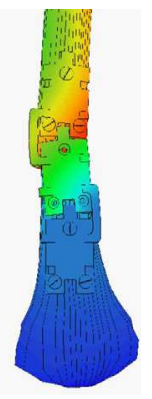

d)

Fig. 18. Two stages of the simulation movie: for the virtual assembly (fractured tibia and modular plate): a) the von Mises stress diagrams [Pa]; b) the displacement diagrams [mm];

The human femur osteosynthesis process using modular adaptive plates based on shape memory alloys can be numericaly simulated with the help of ANSYS software packages, following 3 steps. Used materials: Cortical bone: E=18000 MPa, Poisson's Coefficient=0,3; Spongious bone: E=50 MPa, Poisson's Coefficient=0,25; Plates: (Titanium); Fixing screws (Titanium). The holding elements: Nitinol - simulated material in ANSYS using the material model "shape memory alloy".To highlight the use of nitinol for the holding elements it is necessary to follow three steps.

For the simulation of the nitinol elements behavior and for the study of their effects, we have considered only the surface placed in the proximity of the humeral head. The small plates were placed both ways of the longitudinal axis of the bone, proximate under its head, following the curve and dip of the bone surface geometry. There were simulated the screws for fixing the small plates and the bone. On a bone area situated on the region of the intermediate plates the bone was interrupted (on a distance of 1-2 $\mathrm{mm}$ ), obtaining two bone segments that are about to be joined using the small plates and the nitinol holding elements. The plates are not fixed in an initial position, they can move $2 \mathrm{~mm}$. The stress and 
displacements diagrams for bone, for plate modules and for staples, for each of the three process steps are obtained.

Step 1. The upper and lower plates are fixed with screws on the bone. It simulates the mounting of head off for holding elements on the fixed plates on the bone, the holding elements having the other head already mounted in the middle plates (common nodes). The temperature of all the elements and of the holding elements is $23^{\circ} \mathrm{C}$. Resultant displacements in plate modules and resultant displacements in femur bone are presented.

Step 2. The ends of the nitinol elements are considered mounted in plates, considering the pretension of step 1, eliminating imposed movements, and realizing the state of tension for mounting the implant.

Step 3. Starting from the final state of tension obtained in step 2 we are simulating the increase of temperature for holding elements from room temperature to body temperature $36.5^{\circ} \mathrm{C}$. Resultant displacements in plate modules, Von Mises stress in Nitinol staples and resultant displacements in femur bone are presented.

The use of nitinol elements makes contact pressure between the two bone segments to grow by $58 \%$. The values of maximum tensions on the plates and on the fixing screws are placed below the limit of proportionality.

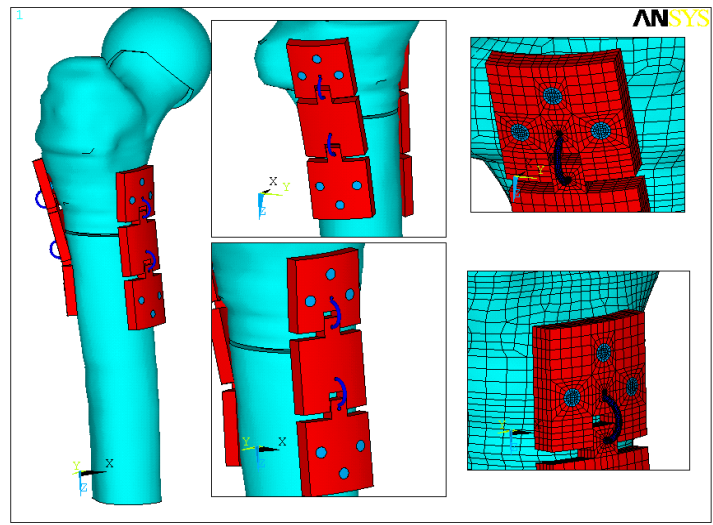

Fig. 19. The finite element model of the femur-implant assembly

Step 1

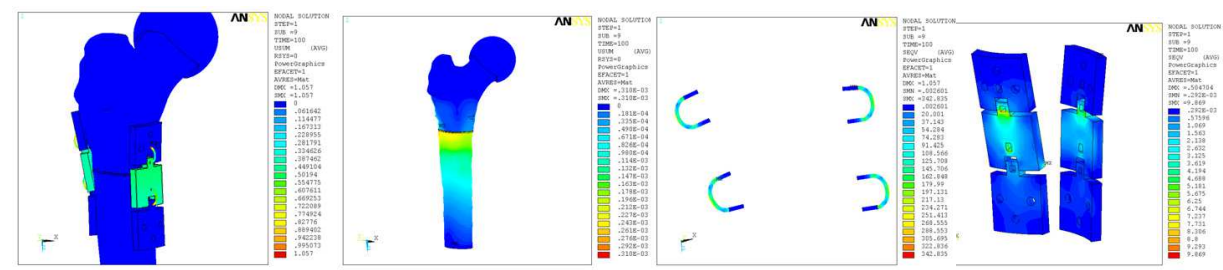

Fig. 20. Total displacements of the second module (a), total displacements for the femur (b), von Mises stress in the element (c), von Mises stress in the plates (d) 
Step 2

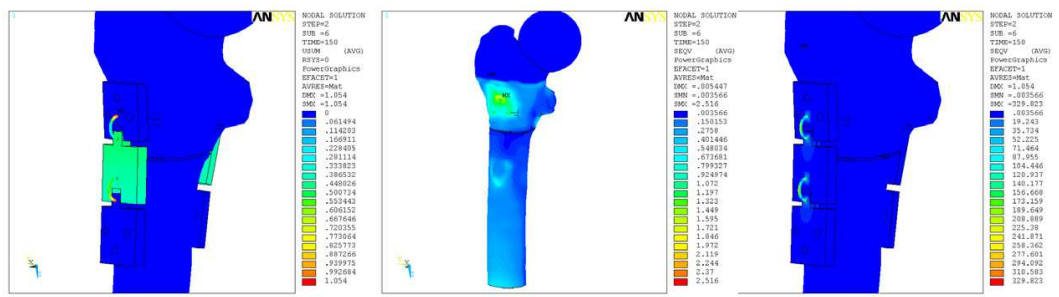

Fig. 21. Total displacements in the bone-implant assembly (a), von Mises stress in femur (b), von Mises in the element (c)

Step 3

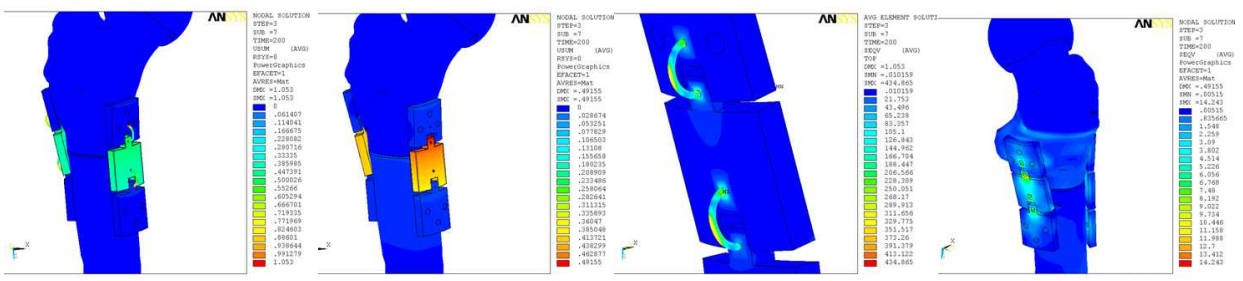

Fig. 22. Total displacements in the bone-implant assembly (a), total displacements in the plates (b), von Mises stress in the element (c) and von Mises stress in the plates (d)

\subsection{Orthopaedic modular centro-medullar rods based on shape memory alloys}

Centro-medullar rods can be used only for diaphyseal fracture fixation of long bones (femur, tibia, and humerus) which limit their use. They make a good centring but compaction is quite poor. When these rods are blocked by passing a proximal screw and a distal one, transversely, trough the bone and rod, it results in the cancellation of compaction forces and implicitly the delay of consolidation, with the development of pseudarthrosis.

Disadvantages of classical centro-medullar rods are that their shape and length do not adapt to the bone channel and that they allow rotation of bone fragments from fractures (the main cause of pseudarthrosis). The rods also get stuck in the medullar canal of the bone and they are difficult to extract after the reduction of the fracture centre and bone healing. If the centro-medullar rod is not well calibrated, it does not prevent rotation of bone fragments and, therefore, does not always permit a good compaction of the fragments, causing pseudoarthrosis. Also in the fracture centre, micro-movements can occur leading to fatigue of the rod's material and, implicitly, to breaking. Centro-medullar rods that have mobility can cause important degenerative-dystrophic injuries at the interface with the fracture centre.

The technical solution consists in designing and execution of a centro-medullar rod whose dimensional characteristics (length and diameter) can be adapted to the medullar canal of the bone.

The total length of the centro-medullar rod can be adjusted by simply substituting the two modules which can be adapted for different bone lengths. Also, two modules may slide 
partially or wholly on the part of the extreme deformation module through the grooves made on these surfaces. The central module is made of a shape memory material which, under the influence of temperature, will deform, allowing the surface of the rod to mold to the medullar canal of the bone.

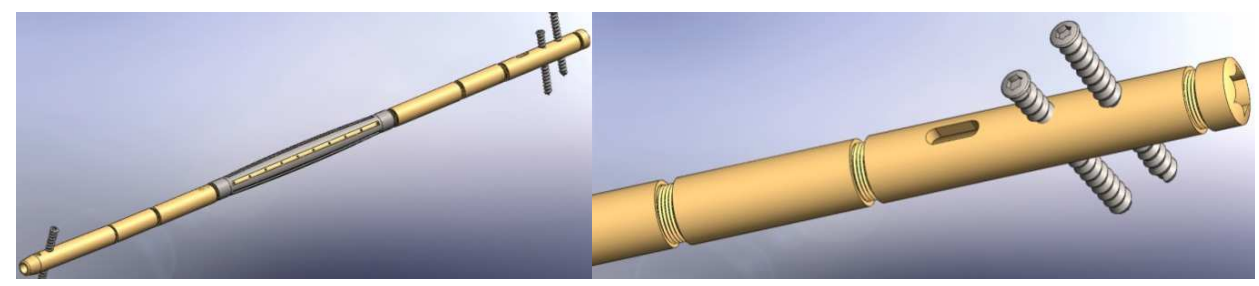

Fig. 23. The first variant of the intramedullary rod system

The second variant: the device is composed of an actuation rod 1 which inserts the steel clips 4 into the bone through the holes made in the modules 2, thus fixing the device into the medullar canal of the bone (in total, the intramedullar rod system has four steel clips). The device is based on the Nitinol module 3 which expands when the intramedullary nail is inserted into the bone (by increasing the temperature to the level of the body temperature). In addition, to better link the device to the medullar canal we use the Nitinol wires 5 which are placed on the distal segment of the device. The modules 2 can slide over the two extreme surfaces of the Nitinol module 3, thus enhancing the versatility of the device (i.e. ensuring various types and dimensions of the bone from one individual to another).
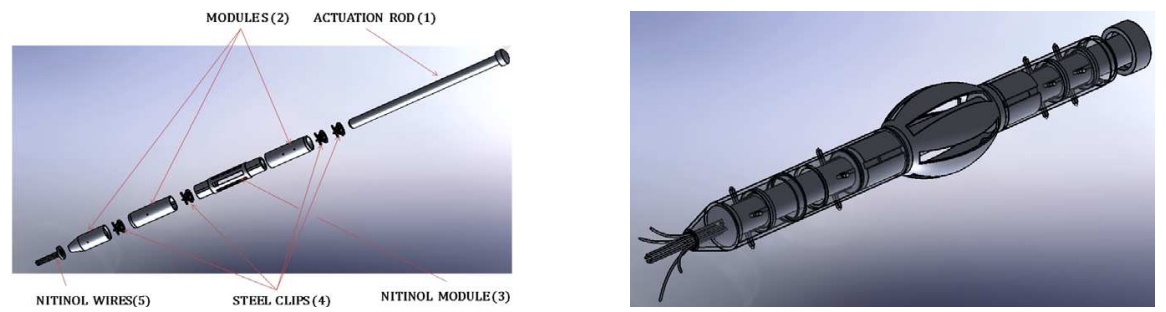

Fig. 24. An exploded view of the intramedullary rod system and the intramedullary nail system in the active state

In the passive state, the Nitinol module and wires are not activated by the rise of the temperature in the human body, the biocompatible steel clips having the legs close together. By contrast, in the active state, the Nitinol module and wires expands and the actuation rod forces the steel clips to penetrate the bone and firmly lock the intramedullary nail to the medullar channel (Fig. 24). The centro-medullar rod based on intelligent materials avoids the disadvantages of conventional centro-medullar rods aforesaid and solves their problems, in that:

- The rod is modular (composed of several components with suitable lengths and diameters which are assembled together) and adaptable to any type of shaft of long bone fracture (shape memory elements are used for a good cohesion between the centro-medullar channel and the centro-medullar rod), 
- Easy to manufacture thanks to components with simple shapes, most components having two threaded surfaces which are used to assemble the next components.

- $\quad$ Easy to extract by cooling the shape memory material

- Provide good compaction of the bone fragments, lowering or eliminating the risk of non-union (pseudo-arthrosys);

- does not allow micro-movements between bone fragments found in fracture centre

- Motion stability is ensured by continuous inter-fragmentary compression

- Avoid the appearance of important degenerative-dystrophic lesions on the contact surface of the fracture centre.

\subsection{External fixator actuated by shape memory alloys elements}

In the open fractures with important coetaneous lesions (type III) using the osteosynthesis materials (plates, centro-medullar rods) is a real danger for infection. In these cases one can use the external fixator which comprises threaded rods or Kirschner brooch which are fixated in the bone fragments at a certain distance above and below the fracture centre, passing through the healthy tissue. These structures are linked externally with rods or circles. In the case of an external fixator, the resistance required to stabilize and consolidate the fracture changes in time, the initial fixation must be rigid enough in order to withstand the mechanical stress that appear once the patient can walk, without fracture disequilibrium. In the same time, the fixator rigidity has to be under certain limits in order to allow the development of pressures at the fracture centre which stimulate the callus formation. In order to obtain the highest resistance for the fixator, several requests must be fulfilled: the distance between the rod and bone to be reduced, the pins diameter to be augmented, the pins located near fracture to be close one to other, the pins thread to be totally inserted in the bone.
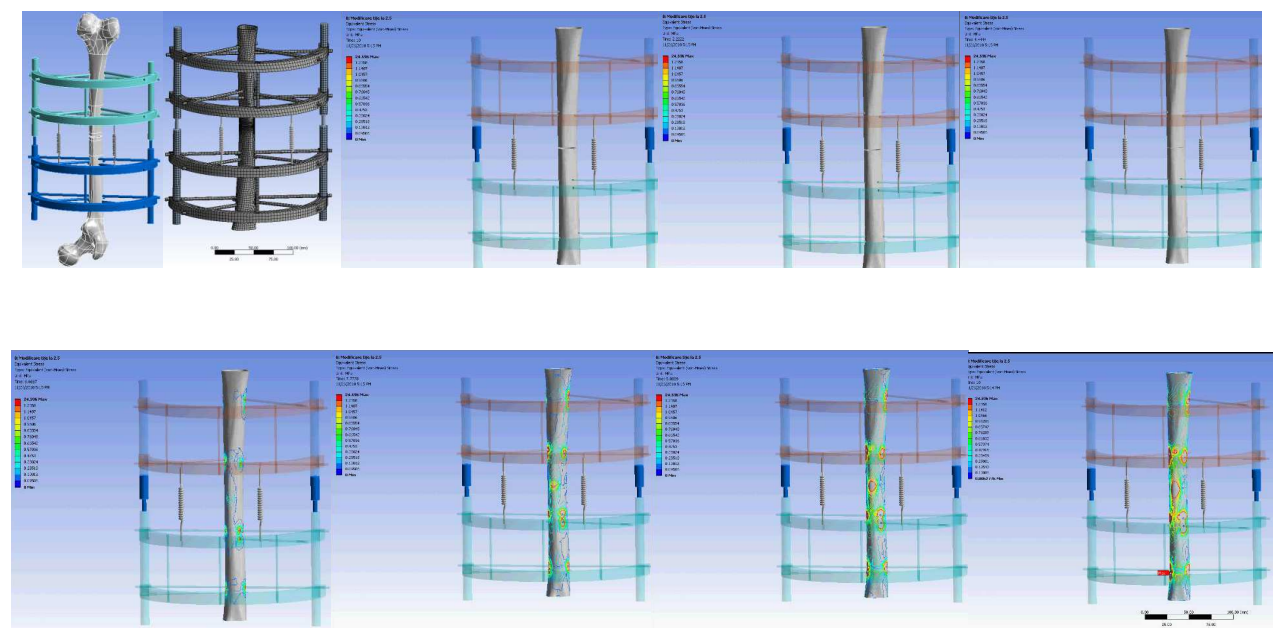

Fig. 25. Sequential frames of the femur external fixator - showing the osteosynthesis process 
The management of bone fractures using an external fixator, adjustment of the bone segment is often necessary to reduce residual deformities. Proposed unilateral external retainer is composed of bone pins inserted into the proximal and distal segment, four semicircular frames, two telescopic side rods, and two of Nitinol compression springs that are designed to compact fracture, the effect of compression on bone fragments interested. It was also simulated femoral shaft osteotomy. In addition to studies of adjustability of the retainer, this model is used to investigate the rigidity of the retainer for evaluating device performance. Transverse fracture was simulated on the axis of the femur and bone segments were modelled as rigid. Were analyzed several cases of bone fragment alignment study using ANSYS software, based on finite Elements Method. Different sequences are generated.This is an example of the need for practical study and application of clinically relevant biomechanical analysis of the results.
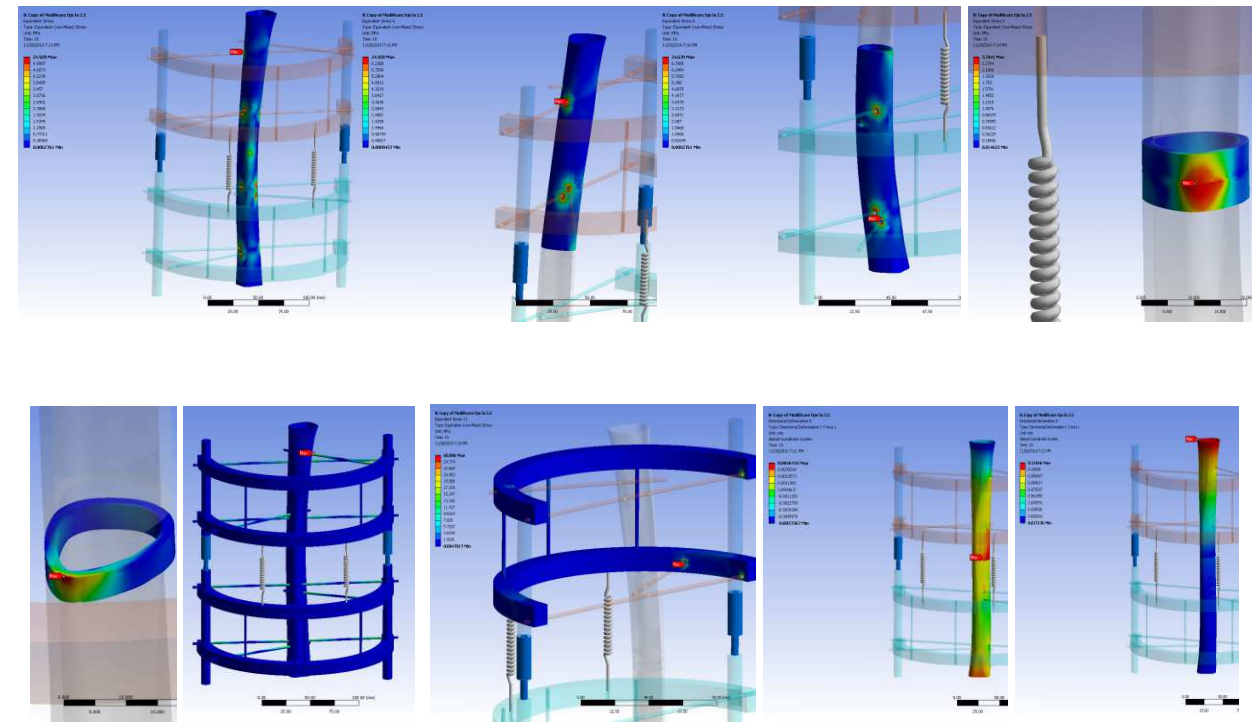

Fig. 26. Stress and deformation maps recorded in the femur-external fixator assembly, when the NiTi springs are placed towards the symmetry axis of the assembly

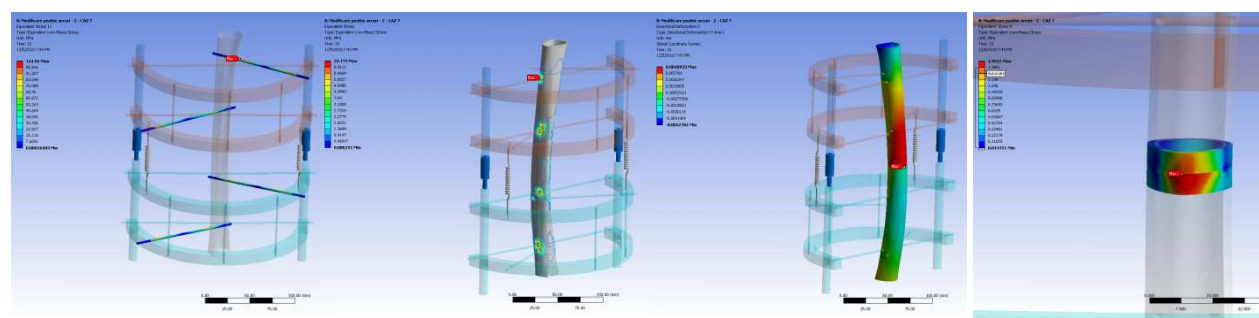

Fig. 27. Stress and deformation maps in the femur-external fixator assembly, the case when NiTi springs are placed towards the lateral rods of the fixator 


\subsection{Orthopaedic implants used for osteoporotic bones fractures}

Osteoporosis is a disease which leads to the reduction of the bone minerals and is directly related to the age of the patient. Therefore, osteoporosis can cause fractures to the spine and to the femur extremities and, especially, to the humeral head. In comparison to the patients who have normal bone density, patients with osteoporosis can suffer fractures of the spine or long bones from low magnitude forces or minor trauma. The most frequent compression fractures of the osteoporotic spine are located at the thorax and lumbar vertebrae level. These fractures can cause acute pain of the back at the level of the fractured vertebra. Once a spinal fracture caused by osteoporosis has occurred the risk of another is increased fourfold compared to the case of non-osteoporotic patients.
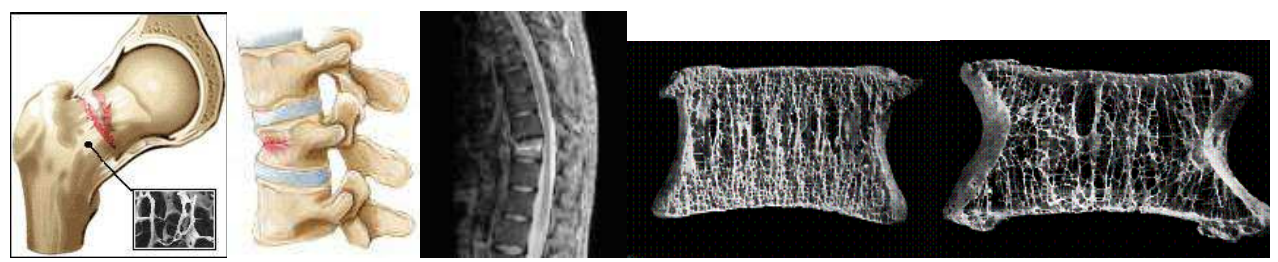

Fig. 28. The humeral head and vertebra fracture and the structure of the normal bone and osteoporotic spongy bone

The numerical simulation of osteoporosis in the case of the femoral head is based on the hypothesis according to which the effect of the osteoporosis is equivalent to the change in the mechanical characteristics of the bone, more exactly, of the osteoporotic spongy tissue. These mechanical characteristics are the longitudinal elasticity module (E) and the Poisson coefficient. In the case of the bone structure we have considered the material as orthotropic, having different values of the mechanical characteristics on the $\mathrm{Ox}, \mathrm{Oy}$ and $\mathrm{Oz}$ axis. The purpose of this simulation is to visualize the influence of osteoporosis in the whole mass of the bone and spongy tissue in order to draw some conclusions regarding:

- the most dangerous zones in which the mechanical properties are diminished;

- $\quad$ the degree of osteoporosis at which the bone cannot support the loads;

- the influence of osteoporosis on the mechanical resistance of the bone.

From the presented stresses maps one can observe that the resistance of the bone and, especially, of the spongy tissue drops by $50 \%$ for a degree of osteoporosis of $15 \%$, therefore at $20 \%$ osteoporosis the fractures of the humeral head are imminent. One can observe also that the most dangerous zone is located at the neck of the humeral head where, in fact, the fractures occur frequently.
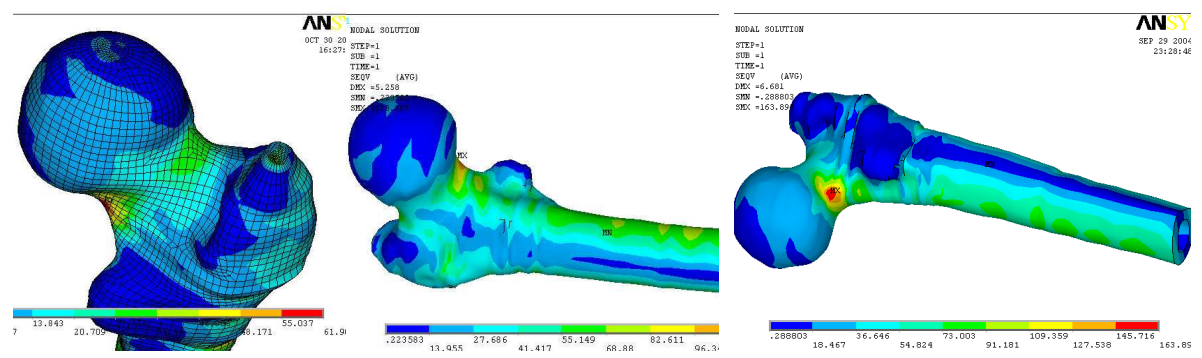

Fig. 29. Von Misses stresses for $0 \%, 15 \%$, 20\% osteoporotic bone 
The proposed plate used for the fractures of the osteoporotic bones has two lateral legs with divergent longitudinal directions one to the other, having sharp heads and fixing devices and also a transversal arm which connects the two legs with a configuration which presents a spatial curvature, with a convex profile. The plate is designed in such a way that the lateral legs are different in shape in relation to the way the metaphisis is penetrated or to the implantation in the cortical bone. The lateral legs are united through a transversal arm by connection zones internally and externally, conveniently curved to obtain a better elasticity of the lateral legs and, more specifically, of the structure of the plate as a unit. The lateral legs have on their inner surface a series of teeth directed to the interior of the transversal arm and at the end of the inner surface of each one of the lateral legs one can observe the conical surfaces directed outwardly. This fact constrains the surgeon to augment the distance between the lateral legs during the implantation of the osteoporotic plate. The inner surface of the transversal arm presents an augmented rugosity in order to allow micro-vascularisation and, therefore, cortical callus formation. Moreover, in the case of lower limb bone osteotomy, the implant must be inserted on the longitudinal axis of the bone. In conceiving this implant we established a convex profile of the transversal arm of the plate with the same curvature as the bone. The advantage of added elasticity to the lateral legs is important because, when the human body temperature is attained, they exert compression force on the bone fragments and amplify the automatic effect of retaining the bone fragments on all fracture sides. The proposed implant for the osteoporotic bone can be perfectly implanted into the bone, offering a strong holding effect of the bone fragments on each side of the fracture line.
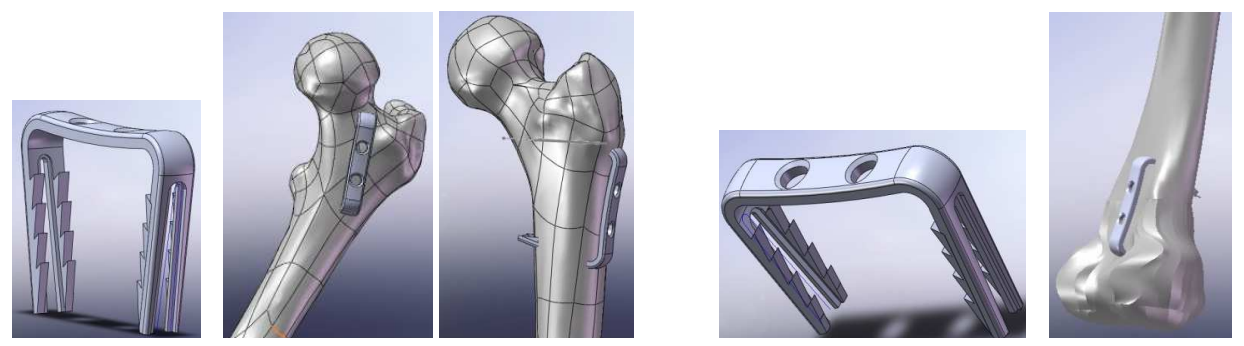

Fig. 30. The plate is implanted in the proximal epiphysis of the femur (first variant), the plate is implanted in the distal epiphysis of the femur (second variant)

\subsection{Modular adaptive orthopaedic network based on shape memory alloys}

The problem which this modular-adaptive implant proposal solves is that the implant ensures a modular adaptive network to the fracture due to the properties of the material from which the network is made (Nitinol), an elastic coupling and the stimulation of the rehabilitation of the bone continuity. As a function of the severity and particularity of the case, in the central area of all modules that form the network or just in the case of a small number of modules the simulative corresponding drugs can be stored. The central area can be perforated by the surgeon so the drugs can be administrated locally, in the traumatised area, the flow depending on the dimension of the penetrating needle. The smart material (for example Nitinol) that makes the modular network is characterised by superelasticty similar to the bone structures, a good image revealed by radiological investigations and a good physical and chemical compatibility which can be assimilated by an augmented resistance to the electrolytic effect of the biological environment. Due to the pseudoelastic property of shape memory alloys, even 
when resorption occurs between the two fragments, the implant maintains its compressive effect, which has a positive influence on fracture healing.

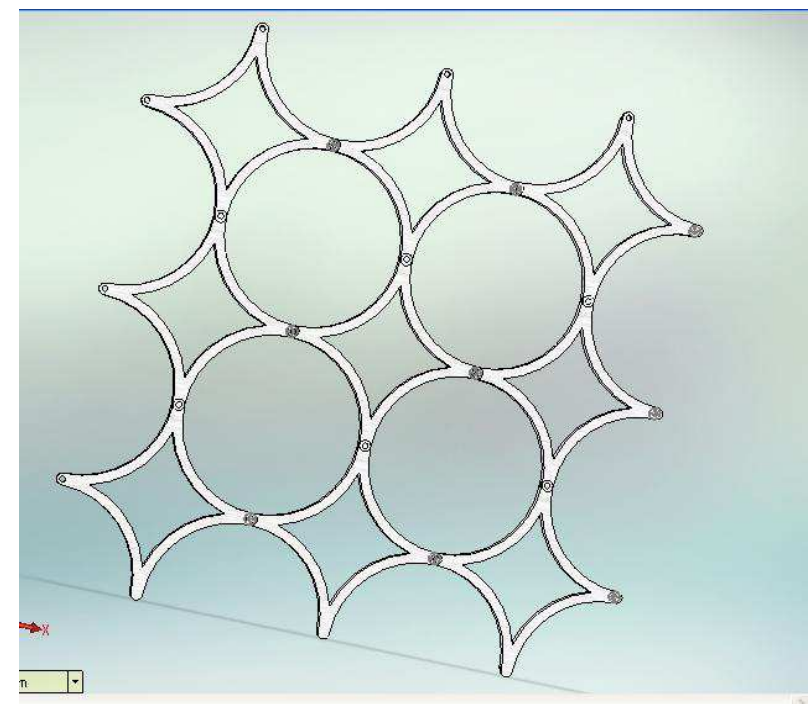

Fig. 31. The schema of the modular adaptive orthopaedic network
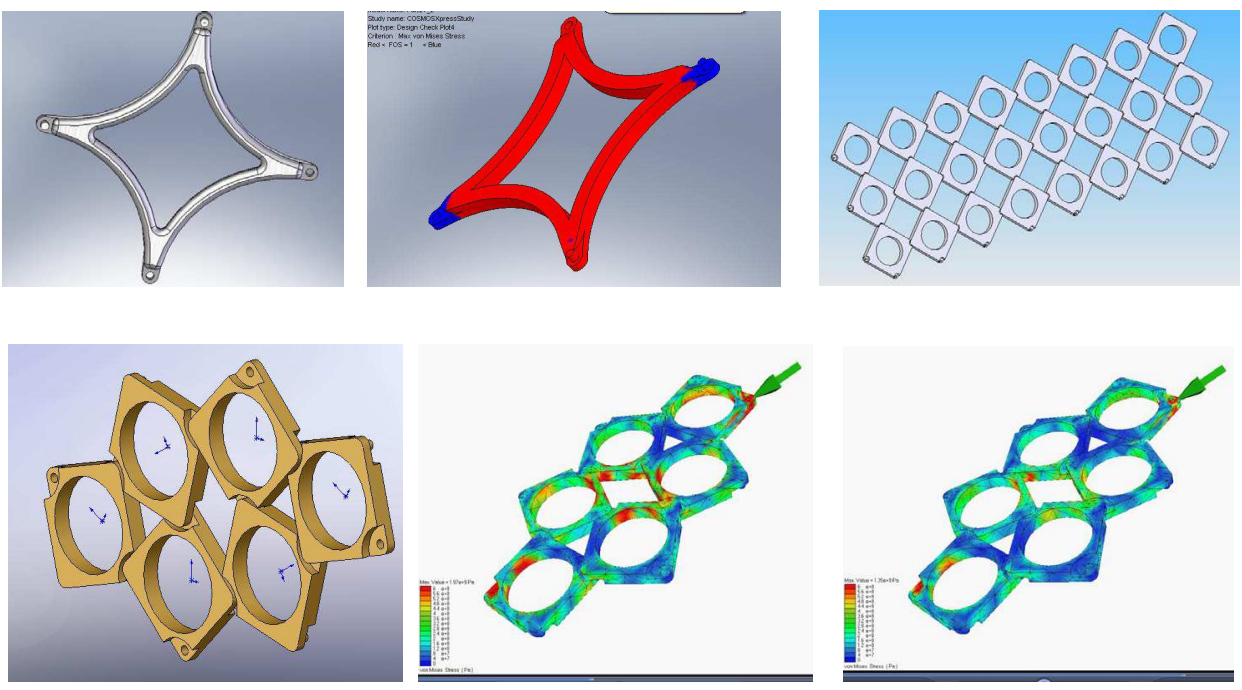

Fig. 32. For a force given by equation $F=-800^{*} \sin (6.282 *$ time) on the $\mathrm{OX}$ axis we present the stress maps corresponding to the network in two different moments of the dinamic load 
A comparison between classical implants and proposed modular implants based on shape memory alloys is presented:

\begin{tabular}{|l|l|}
\hline Traditional implants & Proposed implants \\
\hline $\begin{array}{l}\text { Big dimensions, configuration } \\
\text { which results in the redundant bone } \\
\text { callus }\end{array}$ & $\begin{array}{l}\text { Small dimensions, completely adaptable to } \\
\text { the fracture }\end{array}$ \\
\hline $\begin{array}{l}\text { Invasive surgical interventions in order } \\
\text { to couple the implants }\end{array}$ & $\begin{array}{l}\text { Minimal invasive surgical techniques are } \\
\text { used for this types of implants - micro- } \\
\text { incisions }\end{array}$ \\
\hline $\begin{array}{l}\text { Medium risk of postsurgical infections } \\
\text { due to the surgical intervention }\end{array}$ & $\begin{array}{l}\text { Minimal risk due to reduced area of surgical } \\
\text { intervention }\end{array}$ \\
\hline $\begin{array}{l}\text { Neutral from the point of view of the } \\
\text { bio-stimulation bone restoration }\end{array}$ & $\begin{array}{l}\text { Bio-stimulation properties for bone growth, } \\
\text { reduced time for healing the fracture zone }\end{array}$ \\
\hline $\begin{array}{l}\text { Require an important number of } \\
\text { physical connections (holes) for implant } \\
\text { fixation }\end{array}$ & $\begin{array}{l}\text { Small number and reduced dimensions for } \\
\text { the fixation holes (in some cases the number } \\
\text { of holes can be zero) }\end{array}$ \\
\hline & $\begin{array}{l}\text { Due to the constant pressure that the } \\
\text { implants make the fracture fragments are } \\
\text { well compacted, no micro-movements are } \\
\text { The fixation problems related to classical } \\
\text { implants can cause pseudo arthritis } \\
\text { arthritis }\end{array}$ \\
\hline
\end{tabular}

\section{Theoretical and experimental studies for NiTi staples}

\subsection{Decomposition of the elasticity matrix for Nitinol structure phases}

The form of the elasticity matrix contains the restrictions done by the symmetry theory of classical crystallography and it permits a simple geometrical interpretation of the relationship between stress and strain regardless of the degree on anisotropy. These restrictions are reflected in the invariant structures of the spectral decompositions. The spectral forms are determined by the symmetry groups, and are independent of the values of the elastic constants. In (Cowin \& Mehrabadi, 1987, 1990) the eigenvalues and eigenvectors for anisotropic elasticity were determined. (Ting, 1987) has discussed the eigenvalue problem in connection with his study of the invariants of the elasticity tensor. The first spectral decomposition of the elasticity tensor was made in (Rychlewski \& Zhang, 1989), using tensorial products. Then, (Sutcliffe, 1992) developed this method and they used it for different types of symmetries. A more simple method, using matrix $6 \times 6$ was used in (Theocaris \& Philippidis, 1989) for the decomposition of the rigidity matrix of the transversal isotropic materials.

In the case of the linear-elastic materials, the dependence between the deformation matrix components and the stress matrix components can be written as a linear dependence:

$$
\sigma_{i j}=\sum_{k=1}^{3} \sum_{l=1}^{3} S_{i j k l} \varepsilon_{k l}
$$

This dependence can be written on the following form:

$$
(\sigma)=[S](\varepsilon)
$$

where: 


$$
\begin{gathered}
(\varepsilon)=\left\{\begin{array}{c}
\varepsilon_{11} \\
\varepsilon_{22} \\
\varepsilon_{33} \\
\sqrt{2} \varepsilon_{23} \\
\sqrt{2} \varepsilon_{13} \\
\sqrt{2} \varepsilon_{12}
\end{array}\right\} ; \quad(\sigma)=\left\{\begin{array}{c}
\sigma_{11} \\
\sigma_{22} \\
\sigma_{33} \\
\sqrt{2} \sigma_{23} \\
\sqrt{2} \sigma_{13} \\
\sqrt{2} \sigma_{12}
\end{array}\right\} \\
{[S]=\left[\begin{array}{cccccc}
S_{1111} & S_{1122} & S_{1133} & \sqrt{2} S_{1123} & \sqrt{2} S_{1113} & \sqrt{2} S_{1112} \\
S_{2211} & S_{2222} & S_{2233} & \sqrt{2} S_{2223} & \sqrt{2} S_{2213} & \sqrt{2} S_{2212} \\
S_{3311} & S_{3322} & S_{3333} & \sqrt{2} S_{3323} & \sqrt{2} S_{3313} & \sqrt{2} S_{3312} \\
\sqrt{2} S_{2311} & \sqrt{2} S_{2322} & \sqrt{2} S_{2333} & 2 S_{2323} & 2 S_{2313} & 2 S_{2312} \\
\sqrt{2} S_{1311} & \sqrt{2} S_{1322} & \sqrt{2} S_{1333} & 2 S_{1323} & 2 S_{1313} & 2 S_{1312} \\
\sqrt{2} S_{1211} & \sqrt{2} S_{1222} & \sqrt{2} S_{1233} & 2 S_{1223} & 2 S_{1213} & 2 S_{1212}
\end{array}\right]}
\end{gathered}
$$

with:

$$
S_{i j k l}=S_{j i k l}=S_{i j l k}=S_{k l i j}
$$

Basically, SMA presents two well-defined crystallographic phases, i.e., austenite and martensite. Martensite is a phase that is easily deformed, reaching large strains $(\sim 8 \%)$, and in the absence of stress, is stable only at low temperatures; in addition, it can be induced by either stress or temperature. The kinematics associated with the martensitic phase transformation in a single crystal is described for a cubic to tetragonal and cubic to monoclinic transformation, and the lattice invariant strain by plastic slip is discussed (Patoor et al.,2006). When the martensitic transformation takes place, numerous physical properties are modified. During the transformation, a latent heat associated with the transformation is absorbed or released based on the transformation direction. The forward, austenite-tomartensite transformation is accompanied by the release of heat corresponding to a change in the transformation enthalpy (exothermic phase transformation). The reverse, martensiteto-austenite transformation is an endothermic phase transformation accompanied by absorption of thermal energy. For a given temperature, the amount of heat is proportional to the volume fraction of the transformed material.

\subsection{Symmetry cases of Nitinol crystallographic phases}

We present the elasticity matrix for the crystallographic phases of Nitinol. For the trigonal crystallographic structure, the matrix [S] has the expression:

$$
[S]=\left[\begin{array}{cccccc}
C_{11} & C_{12} & C_{13} & 0 & -\sqrt{2} C_{15} & 0 \\
C_{12} & C_{11} & C_{13} & 0 & \sqrt{2} C_{15} & 0 \\
C_{13} & C_{13} & C_{33} & 0 & 0 & 0 \\
0 & 0 & 0 & C_{44} & 0 & 2 C_{15} \\
-\sqrt{2} C_{15} & \sqrt{2} C_{15} & 0 & 0 & C_{44} & 0 \\
0 & 0 & 0 & 2 C_{15} & 0 & C_{11}-C_{12}
\end{array}\right]
$$


In this case, the eigenvalues are:

$$
\begin{aligned}
& \lambda_{1}=\frac{1}{2}\left[C_{11}+C_{12}+C_{33}+\sqrt{\left(C_{11}+C_{12}-C_{33}\right)^{2}+8 C_{13}^{2}}\right] \\
& \lambda_{2}=\frac{1}{2}\left[C_{11}+C_{12}+C_{33}-\sqrt{\left(C_{11}+C_{12}-C_{33}\right)^{2}+8 C_{13}^{2}}\right] \\
& \lambda_{3}=\lambda_{6}=\frac{1}{2}\left[C_{11}-C_{12}+C_{44}+\sqrt{\left(C_{11}-C_{12}-C_{44}\right)^{2}+16 C_{15}^{2}}\right] \\
& \lambda_{4}=\lambda_{5}=\frac{1}{2}\left[C_{11}-C_{12}+C_{44}-\sqrt{\left(C_{11}-C_{12}-C_{44}\right)^{2}+16 C_{15}^{2}}\right]
\end{aligned}
$$

and the matrix of eigenvectors is:

$$
[\mathrm{X}]=\left[\begin{array}{cccccc}
\frac{1}{\sqrt{2}} \sin \alpha & -\frac{1}{\sqrt{2}} \cos \alpha & \frac{1}{\sqrt{2}} \cos \beta & 0 & \frac{1}{\sqrt{2}} \sin \beta & 0 \\
\frac{1}{\sqrt{2}} \sin \alpha & -\frac{1}{\sqrt{2}} \cos \alpha & -\frac{1}{\sqrt{2}} \cos \beta & 0 & -\frac{1}{\sqrt{2}} \sin \beta & 0 \\
\cos \alpha & \sin \alpha & 0 & 0 & 0 & 0 \\
0 & 0 & 0 & \cos \beta & 0 & \sin \beta \\
0 & 0 & -\sin \beta & 0 & \cos \beta & 0 \\
0 & 0 & 0 & -\sin \beta & 0 & \cos \beta
\end{array}\right]
$$

where:

$$
\begin{aligned}
& \sin \alpha=\frac{\left|\lambda_{2}-C_{11}-C_{12}\right|}{\sqrt{2 C_{13}^{2}+\left(\lambda_{2}-C_{11}-C_{12}\right)^{2}}} ; \cos \alpha=\frac{\left|\lambda_{1}-C_{11}-C_{12}\right|}{\sqrt{2 C_{13}^{2}+\left(\lambda_{1}-C_{11}-C_{12}\right)^{2}}} \\
& \sin \beta=\frac{\left|C_{11}-C_{12}-\lambda_{3}\right|}{\sqrt{\left(C_{11}-C_{12}-\lambda_{3}\right)^{2}+4 C_{15}^{2}}} ; \cos \beta=\frac{\left|C_{11}-C_{12}-\lambda_{4}\right|}{\sqrt{\left(C_{11}-C_{12}-\lambda_{4}\right)^{2}+4 C_{15}^{2}}}
\end{aligned}
$$

Particular cases:

a. In the case of the cubic crystallographic structure:

$$
\begin{gathered}
C_{12}=C_{13} ; \quad C_{33}=C_{11} ; C_{44}=C_{11}-C_{12} ; C_{15}=0 \\
{[S]=\left[\begin{array}{cccccc}
C_{11} & C_{12} & C_{12} & 0 & 0 & 0 \\
C_{12} & C_{11} & C_{12} & 0 & 0 & 0 \\
C_{12} & C_{12} & C_{11} & 0 & 0 & 0 \\
0 & 0 & 0 & C_{44} & 0 & 0 \\
0 & 0 & 0 & 0 & C_{44} & 0 \\
0 & 0 & 0 & 0 & 0 & C_{44}
\end{array}\right]}
\end{gathered}
$$

The eigenvalues are:

$$
\lambda_{1}=C_{11}+2 C_{12} ; \lambda_{2}=\lambda_{3}=C_{11}-C_{12} ; \lambda_{4}=\lambda_{5}=\lambda_{6}=C_{44}
$$


The matrix of eigenvectors is:

$$
[X]=\left[\begin{array}{cccccc}
1 / \sqrt{3} & -1 / \sqrt{6} & 1 / \sqrt{2} & 0 & 0 & 0 \\
1 / \sqrt{3} & -1 / \sqrt{6} & -1 / \sqrt{2} & 0 & 0 & 0 \\
1 / \sqrt{3} & 2 / \sqrt{6} & 0 & 0 & 0 & 0 \\
0 & 0 & 0 & 1 & 0 & 0 \\
0 & 0 & 0 & 0 & 1 & 0 \\
0 & 0 & 0 & 0 & 0 & 1
\end{array}\right]
$$

b. In the case of monoclinic crystallographic structure:

$$
[S]=\left[\begin{array}{cccccc}
\mathrm{C}_{11} & \mathrm{C}_{12} & \mathrm{C}_{13} & 0 & 0 & \mathrm{C}_{16} \\
\mathrm{C}_{12} & \mathrm{C}_{22} & \mathrm{C}_{23} & 0 & 0 & \mathrm{C}_{26} \\
\mathrm{C}_{13} & \mathrm{C}_{23} & \mathrm{C}_{33} & 0 & 0 & \mathrm{C}_{36} \\
0 & 0 & 0 & \mathrm{C}_{44} & \mathrm{C}_{45} & 0 \\
0 & 0 & 0 & \mathrm{C}_{45} & \mathrm{C}_{55} & 0 \\
\mathrm{C}_{16} & \mathrm{C}_{26} & \mathrm{C}_{36} & 0 & 0 & \mathrm{C}_{66}
\end{array}\right]
$$

The eigenvalues are:

$$
\lambda_{4}=\frac{1}{2}\left[C_{44}+C_{55}+\sqrt{\left(C_{44}+C_{55}\right)^{2}+4 C_{45}^{2}}\right] ; \quad \lambda_{5}=\frac{1}{2}\left[C_{44}+C_{55}-\sqrt{\left(C_{44}+C_{55}\right)^{2}+4 C_{45}^{2}}\right]
$$

and $\lambda_{1}, \lambda_{2}, \lambda_{3}, \lambda_{4}$ are the roots of the equation:

$$
\lambda^{4}-\mathrm{I}_{1} \lambda^{3}+\mathrm{I}_{2} \lambda^{2}-\mathrm{I}_{3} \lambda+\mathrm{I}_{4}=0
$$

where $\mathrm{I}_{\mathrm{k}}$ is the sum of the diagonal minors of $\mathrm{k}$ degree obtained by cutting the fourth and the fifth columns and rows in matrix [S].

c. In the case of orthorhombic crystallographic structure:

$$
[S]=\left[\begin{array}{cccccc}
C_{11} & C_{12} & C_{13} & 0 & 0 & 0 \\
C_{12} & C_{22} & C_{23} & 0 & 0 & 0 \\
C_{13} & C_{23} & C_{33} & 0 & 0 & 0 \\
0 & 0 & 0 & C_{44} & 0 & 0 \\
0 & 0 & 0 & 0 & C_{55} & 0 \\
0 & 0 & 0 & 0 & 0 & C_{66}
\end{array}\right]
$$

The eigenvalues are:

$$
\lambda_{4}=\mathrm{C}_{44} ; \lambda_{5}=\mathrm{C}_{55} ; \lambda_{6}=\mathrm{C}_{66}
$$

and $\lambda_{1}, \lambda_{2}, \lambda_{3}$ are the roots of the equation:

$$
\lambda^{3}-I_{1}^{\prime} \lambda^{2}+I_{2}^{\prime} \lambda-I_{3}^{\prime}=0
$$


where $I_{k}^{\prime}$ is the sum of the diagonal minors of $\mathrm{k}$ degree obtained by cutting the last three columns and rows in matrix [S].

We can conclude that the eigenvalues depend on the values of the elastic constants, but the eigenvectors are, in part, independent of the values of the elastic constants

\subsection{The analytical expression for the staple compression force}

The stress vector can be written:

$$
(\sigma)=\sum_{i}\left(\sigma_{i}\right)=\sum\left[E_{i}\right] \cdot(\sigma)
$$

Therefore, the specific deformation energy is:

$$
U=\frac{1}{2} \cdot \sum_{(i)} \frac{1}{\lambda_{i}} \cdot\left(\sigma_{i}\right)^{t} \cdot\left(\sigma_{i}\right)
$$

The orthopedic staple can be modeled as a bar which has an initial shape. When temperature increases the staple suffers deformations, changing its shape. Taking into account mechanical considerations, the deformation can be accepted as being caused by an exterior force which is applied to the free extremity. Thus, an increase of temperature, $\Delta \mathrm{T}$ produces a displacement of the free extremity, $\Delta \mathrm{w}$. The same displacement can be produced by a force $(\Delta \mathrm{P})$ which is applied in the free extremity. In fact, the force $(\Delta \mathrm{P})$ is a force to be applied in the free extremity. The total deformation energy is:

$$
U=\frac{1}{2} \cdot \sum_{(i)} \frac{1}{\lambda_{i}} \cdot \iiint_{(D)}\left(\sigma_{i}\right)^{t} \cdot\left(\sigma_{i}\right) \cdot d v
$$

According to the Castigliano theorem energy, the derived strains of an elastic body compared with force value $\Delta \mathrm{P}$ are similar to the displacement projection of the application point of the direction force (fig.1).

$$
\Delta w=\frac{\partial U}{\partial(\Delta P)}
$$

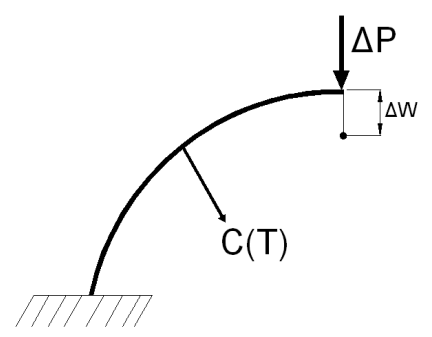

Fig. 35. The loading schema for the Nitinol staple

In the case of small deformations we can accept that the stresses developed in the staple are proportional to the variation force $\Delta \mathrm{P}$. In these conditions, we can write: 


$$
\Delta w=\Delta P \cdot \sum_{(i)} \frac{1}{\lambda_{i}} \cdot \iiint_{(D)}\left(e_{i}\right)^{t} \cdot\left(e_{i}\right) \cdot d v
$$

where:

$$
\left(e_{i}\right)=\frac{\partial\left(\sigma_{i}\right)}{\partial(\Delta P)}
$$

The vectors $\left(\mathrm{e}_{\mathrm{i}}\right)$ depend only on the staple shape. We call $\mathrm{J}_{\mathrm{i}}$ the triple integrals which occur in the relation (24) depend on the temperature, but in a measure much smaller than the eigenvalues. Therefore the integrals can be considered constants. In this case, by passing to the limit, the relation becomes:

$$
\dot{w}=\dot{P} \cdot \sum_{(i)} \frac{1}{\lambda_{i}} \cdot J_{i}
$$

The temperature variation on time is:

$$
T=T_{e}-\left(T_{e}-T_{i}\right) \cdot e^{-c \cdot\left(t-t_{i}\right)}
$$

$T_{i}=$ initially temperature of staple; $t_{i}=$ initially value of time; $c=$ a coefficient which depends on the material thermal conductivity of the staple.

The eigenvalues $\lambda_{i}$ of the elasticity matrix depend on the temperature and on the value $e^{-c\left(t-t_{i}\right)}$. Experimentally, it is demonstrated that the staple deformation is produced with constant speed. In (Kul'kova et al., 1995) were tested three Nitinol wire specimens: a commercially available superelastic $\left(\mathrm{W}_{1}\right)$ wire and two shape memory. It is demonstrated that the dependence of recovery force function on temperature is linear.

Developing in factors series as a function of term $e^{-c\left(t-t_{i}\right)}$ the functions of the proper values and keeping only the first order term, the variation in time of the compression force exerted by the staple is done by:

$$
P=P_{i}+\frac{1}{c} \cdot f\left(T_{e} ; T_{i}\right) \cdot\left(1-e^{-c\left(t-t_{i}\right)}\right)
$$

where: $P_{i}$ is the exerted force at the initial moment $t_{i}$.

\subsection{Experimental studies for the Nitinol staple}

In order to determine the law of variation for the compression force which can be developed by the Nitinol staple as a function of variable environmental temperature and also to validate the compression potential of the staple through constant pressure at the temperature of the human body, we developed an experimental stand (Fig.36).

The experimental stand is made from: -experimental device used to mount the modular adaptive implant; -Spider 8 - a numerical acquisition system, 12 bits resolution, used to measure mechanical parameters, such as: forces, mechanical stresses, pressures, accelerations, velocities, displacements, temperatures.; $-\mathrm{S} 2-100 \mathrm{~N}$ force transducer, $0.1 \%$ linearity, Hottinger type; -FLIR B200 termographic camera; -IBM ThinkPad R5 notebook. 
The Nitinol staple was stored for 15 minutes in $\mathrm{NaCl}$ liquid solution, 30\% concentration, at $20^{\circ} \mathrm{C}$ in a freezer. At this temperature the material of the staple enters in martensitic phase and the lateral pins of the staple are parallel.
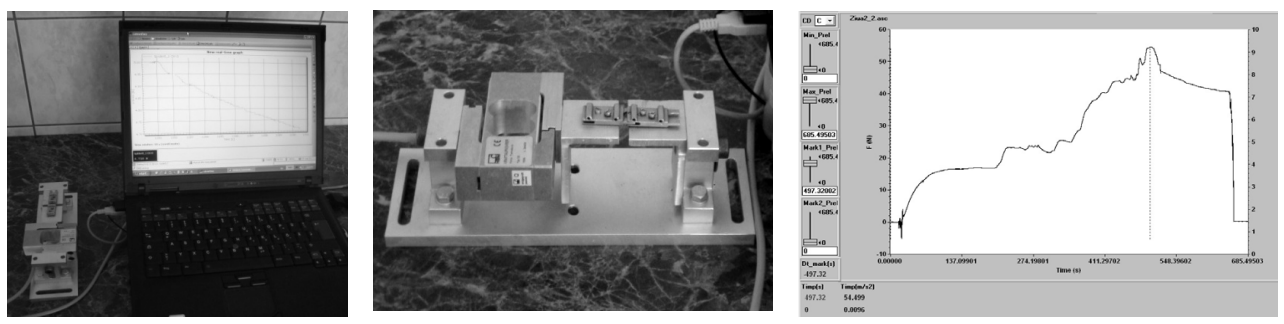

Fig. 36. The experimental stand and the diagram obtained for the staple compression force function on time

Having this shape, the staple was extracted from the $\mathrm{NaCl}$ solution and was easily inserted in special channels of the two implant modules fixed in the device. The staple was then left to attain room temperature $\left(29^{\circ} \mathrm{C}\right)$, thereby compressing the two modules. Afterwards, a jet of hot air was blown onto the staple increasing his temperature in different stages: first, to $31^{\circ} \mathrm{C}$, in a time period of $120 \mathrm{sec}$, then, to $35^{\circ} \mathrm{C}$ in a time period of $120 \mathrm{sec}$ and, finally, to $37^{\circ} \mathrm{C}$, the temperature of the human body. The hot air jet was then stopped and after the staple returned to room temperature it was extracted from the modular implant. Finally, we obtained the force-time diagram (Fig.36). One can observe a maxim value of $54 \mathrm{~N}$ which corresponds to $37^{\circ} \mathrm{C}$ temperature for which the material of the staple entered in the cubic austenite phase. In order to correlate the staple deformation with the developed compression force, the temperature increase has been controlled with the aid of a termographical camera ThermaCam Flir B200. Afterwards, these pictures have been processed and analysed (Fig.37).
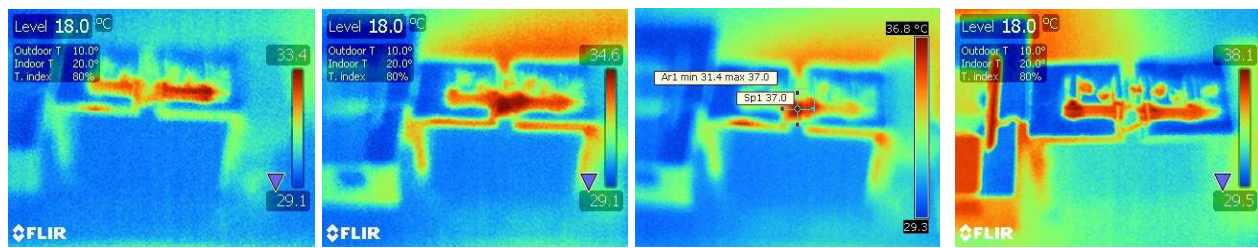

Fig. 37. A few successive images taken with Thermacam

Using the SIMI Motion software, the kinematical parameters of the both extremities of the staple were obtained. In order to link the action mode of the staple and the displacements of the extremity points of the pins during the shape transformation process from the martensitic stage to the austenitic stage, a SIMI Motion acquisition data system was used to obtain the kinematical parameters of the staple. The acquisition data system is composed of: -specialized software; -a Lenovo laptop; -two Sony video cameras (60 frames/sec); -markers.

The main stages of video capture analysis using SimiMotion software are:Camera calibration. 2. Definition of the studied points. 3. Settlement of the points connections. 4. Tracking the points. 5. Extraction of the results. 
By attaching markers, the software automatically generates the equivalent model of the studied system and tracks the displacement of the markers in real time from each frame captured by the video camera, recording and analyzing the positions of the markers which allow us to obtain the motion law. The analysis procedure is based on the attachment of two markers which have been applied on the extremities of the two pins of the staple. A plane was chosen to calibrate the camera, plane given by two axes (OX and OY).

Two successive positions of staple deformation process are presented in Fig.38. In the same figure, the displacement diagram [mm], as functions of time, for the left point is presented. One can be observed that the dependence displacement-time is linear. This observation was used for the determination of the compression force theoretical expression.
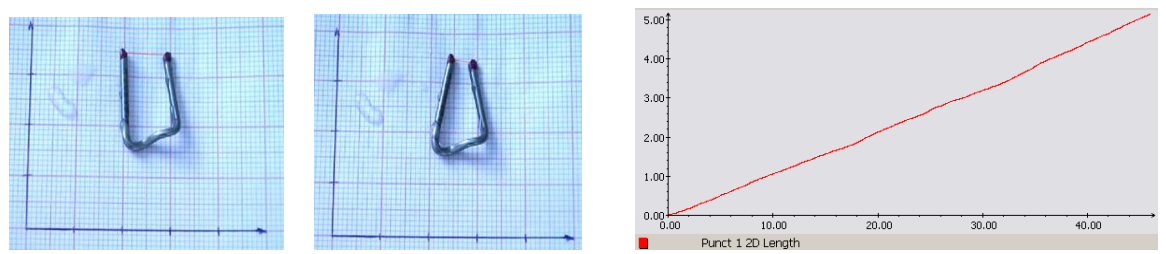

Fig. 38. Two successive positions of staple deformation process and the displacement diagram for the left extremity of the staple

We consider that the staple finishes its deformation when the difference between its temperature and room temperature is $\leq 1^{\circ} \mathrm{C}$. In this hypothesis, the coefficient $\mathrm{c}$ can be determined with the relation:

$$
c=\frac{\ln \left(T_{e}-T_{i}\right)}{\Delta t}
$$

where $\Delta t$ is the staple deformation time.

Experimentally, we can see that $\Delta t=45 \mathrm{sec}$, corresponding to the interval $[-20 ; 29]{ }^{\circ} \mathrm{C}$ of temperature variation (Fig.11). In this case, the resulted value for $\mathrm{c}$ is $0,086 \mathrm{sec}^{-1}$. This value corresponds to the studied staple, so, taking into account the concrete experimental conditions, it is a constant for this product. Any other product made from Nitinol will have other value for c. The experimental diagram presented in fig. 4 shows the stages of the compression force variation corresponding to the stages of the temperature variation (Table 1).

\begin{tabular}{|c|c|c|}
\hline $\begin{array}{c}\text { Temperature } \\
\text { variation }(\circ \mathrm{C})\end{array}$ & $\begin{array}{c}\text { Compression force } \\
\text { variation }(\mathrm{N})\end{array}$ & $\begin{array}{c}\text { Values for } \\
\mathrm{f}\left(\mathrm{T}_{\mathrm{e}}-\mathrm{T}_{\mathrm{i}}\right)(\mathrm{N})\end{array}$ \\
\hline$-20 \ldots . .29$ & $0 \ldots . .17$ & $\mathrm{f}(29 ;-20)=17$ \\
\hline $29 \ldots . .31$ & $17 \ldots .24$ & $\mathrm{f}(31 ; 29)=7$ \\
\hline $31 \ldots \ldots .35$ & $24 \ldots .44$ & $\mathrm{f}(35 ; 31)=20$ \\
\hline $35 \ldots . .37$ & $44 \ldots . .54$ & $\mathrm{f}(37 ; 35)=10$ \\
\hline
\end{tabular}

Table 1. Experimental compression force values

Using the values for $f\left(T_{e}-T_{i}\right)$ as input data in the relation (50), we made a numerical simulation in Maple12 and we obtained the graphic presented in Fig.12. For the numerical simulation, we respected the same temperature increasing stages as in the experimental case. This explains the allure of the numerical graphic. For first temperature increase, from $-20^{\circ} \mathrm{C}$ to $29^{\circ} \mathrm{C}$, the force 
variation is nonlinear, and for the other three stages we observe that the force increasing is less than $10 \mathrm{~N}$ for a temperature increasing with $2^{\circ} \mathrm{C}$, the force variation is linear. For constant temperatures, the force remains constant. The diagram force-displacement proves the maximum of the compression force $(54 \mathrm{~N})$ is obtained in the Nitinol staple at the body temperature, $37^{\circ} \mathrm{C}$. This properties of the Nitinol staple allows its using in orthopedic applications, like simple orthopedic implants, or adaptive modular implants.

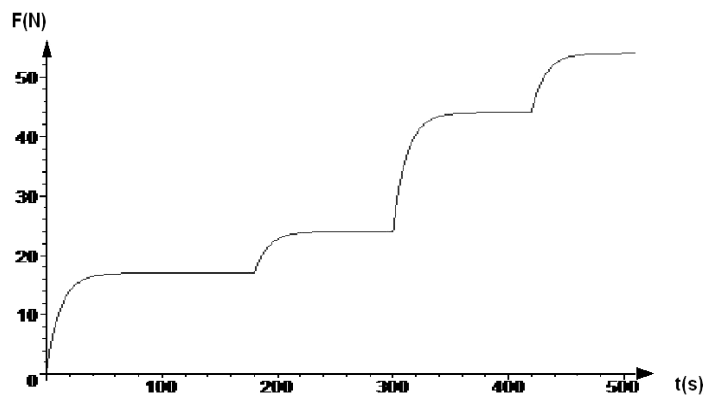

Fig. 39. The compression force-time numerical graphic

\subsection{Electrochemical study on corrosion resistance of Nitinol in physiological media}

The metal materials used as implants must be biocompatible. Biocompatibility means absence of corrosion or allergic reactions. Corrosion is one of the most important processes that affect the functionality and the duration of medical devices made of metals and their alloys used as implants. The failures of the implants were due to significant phenomena of localised corrosion. Corrosion as a test of biocompatibility is a very important factor, which produces metal ions in the biological medium and leads to the degradation of implants. There have been made electrochemical studies "in vitro" in order to determine the corrosion reactions, which are necessary for foreseeing the behaviour of the materials used in implantology. The degradation of metals and alloys in the human body is a combination of effects due to corrosion and mechanical activities. The surface roughness, texture and localized corrosion resistance are the most important characteristics for stabilizing tissueimplant interface. Although several studies have demonstrated the good corrosion resistance and biocompatibility of $\mathrm{NiTi}$, the high nickel content of the alloy ( 55 weight $\% \mathrm{Ni}$ ) and its possible dissolution by corrosion still remains a concern (Ryhänen et al., 1997; Shabalovskaya, 1996; Venugopalan \& Trepanier, 2000; Wever et al., 1998). Tissues in the human body contain water, dissolved oxygen, proteins and various ions, such as chloride and hydroxide, and they present an aggressive environment to metals or alloys used for implantation (Shrier et al., 1995). Corrosion resistance of a metallic implant is thus an important aspect of its biocompatibility (Black, 1992). In addition to the release of ions in the physiological environment, the corrosion process will also result in the deterioration of dimensional parameters of the corroding body (Fontana, 1986). NiTi corrosion behaviour can be significantly improved after specific surface treatments such as electropolishing (Trepanier et al., 1998). In this study the behaviour of Nitinol in physiological serum (PS) and glucose is discussed according to electrochemical measurements and microscopic images (Samide et al., 2008), which were obtained for the material before and after corrosion tests. 


\subsection{Experimental stand}

The Nitinol used had the following composition (weight \%): Ni 49,6\% and $\mathrm{Ti} 50,4 \%$.The samples were degreased with acetone and dried. Physiological serum (PS - $0.9 \% \mathrm{NaCl}$ ) and $5 \%$ glucose were used as the corrosion tests solutions. For the polarization study, a standard corrosion cell with a working electrode made of Nitinol wire with an active surface of $0.314 \mathrm{~cm}^{2}$ was used. The $\mathrm{Ag} / \mathrm{AgCl}$ electrode was used as a reference electrode. The auxiliary electrode was a platinum electrode (surface area- $1 \mathrm{~cm}^{2}$ ). The potentiodinamic polarization was conducted with a scan rate of $20 \mathrm{mVs}^{-1}$, in an electrochemical system, VoltaLab 40, with a personal computer and VoltaMaster 4 software (Figure1). The immersion time of the plates in the respective media was 4 minutes in open circuit, at room temperature. The morphology of the Nitinol surface before and after treatment in the above mentioned solutions was examined using a metallographic microscope Euromex, with Canon camera and included software (Figure 40).
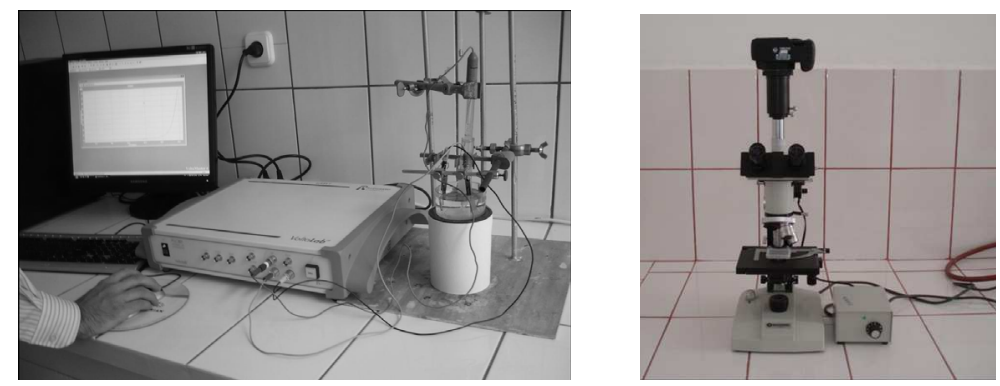

Fig. 40. Electrochemical assembly used for corrosion tests and the metallographic microscope type EUROMEX

\subsection{Electrochemical measurements}

\section{Potentiodinamic curves}

The polarization curves of nitinol wire obtained in physiological serum (PS) and glucose are presented in Fig41.
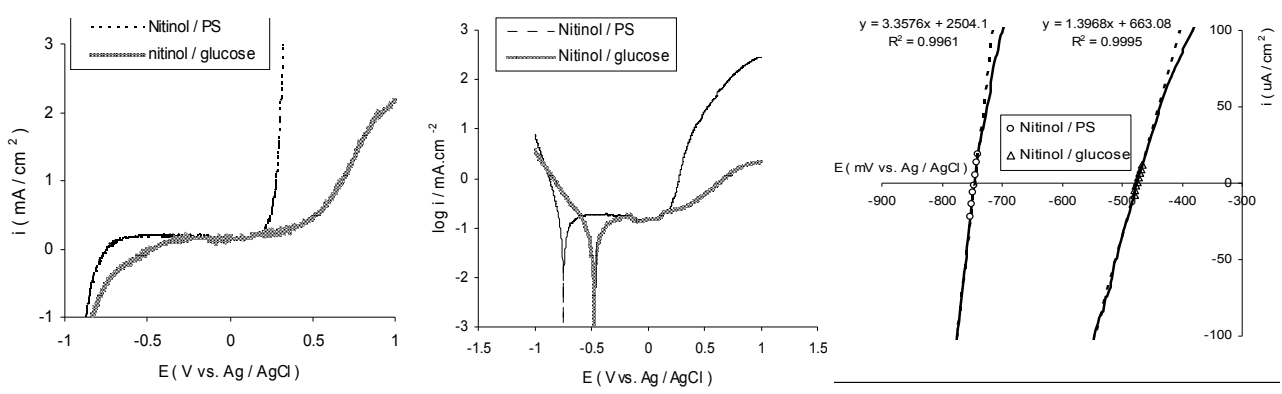

Fig. 41. Polarization curves of Nitinol wire obtained in physiological serum and glucose, after immersion time in open circuit of 2 minutes, at room temperature; Tafel diagram for Nitinol staple corroded in physiological serum and glucose; Polarization resistance $\left(R_{p}\right)$ of Nitinol wire corroded in physiological serum and glucose 
The study of the response Nitinol given by polarizing in physiological serum (PS) and glucose solutions, simulating the tissue fluid conditions indicate that the critical potential in pitting $\left(E_{\mathrm{cp}}\right)$ are shifted to a higher value, when corrosion study of Nitinol in glucose carried out. It has also been observed that the critical potential in pitting decrease from $504 \mathrm{mV}$ in glucose to $246 \mathrm{mV}$ in physiological serum (Table 2).

\begin{tabular}{|l|l|l|l|l|}
\hline Medium & $\begin{array}{l}E_{c p} \\
(\mathrm{mV} / \mathrm{Ag} / \mathrm{AgCl})\end{array}$ & $\begin{array}{l}E_{\text {corr }} \\
(\mathrm{mV} / \mathrm{Ag} / \mathrm{AgCl})\end{array}$ & $\begin{array}{l}\boldsymbol{i}_{\text {corr }} \\
\left(\mu \mathrm{A} \mathrm{cm}^{-2}\right)\end{array}$ & $\begin{array}{l}\boldsymbol{R}_{p} \\
\left(\boldsymbol{\Omega} \mathrm{cm}^{-2}\right)\end{array}$ \\
\hline $\begin{array}{l}\text { Physiological } \\
\text { serum }\end{array}$ & 246 & -746 & 2.3 & 297 \\
\hline Glucose & 504 & -474 & 0.94 & 716 \\
\hline
\end{tabular}

Table 2. Electrochemical parameters obtained for NiTi corroded in physiological serum and glucose, at room temperature

This suggests that glucose acts by adsorption at site on the metal surface and it was formed, more adherent and more uniform passive layer than in physiological serum.

Tafel polarization. In Tafel domain, the polarization curves were performed in the potential range $-1000 \mathrm{mV}$ to $1000 \mathrm{mV}$, with a scan rate of $20 \mathrm{mV} / \mathrm{sec}$. The polarization curves obtained after 2 minutes of immersion are presented in Figure 4. The performed tests showed that:

- glucose leads to a corrosion $\left(E_{\text {corr }}\right)$ potential shifted to more positive values;

- the corrosion potential shifted to more positive values is correlate with a significant corrosion current $\left(i_{\text {corr }}\right)$ decrease;

- glucose solution disturbs significantly cathodic reaction and reduces the anodic reaction in a considerable manner;

- $\quad$ the electrochemical parameters presented in table 1 were calculated using VoltaMaster4 software at smoothing 9, calculi zone 1800 and segment $600 \mathrm{mV}$.

Polarization resistance method-Stern Method. The polarization curves obtained in the potential ranges near to corrosion potentials were recorded with a scan rate of $10 \mathrm{mV} \mathrm{s}^{-1}$. The linearization was accomplished in the domain of over-voltages values $\pm 10 \mathrm{mV}$ (Figure 5). The slopes $(d i / d E)_{E \rightarrow E c o r r}$ of the lines from Figure 5 represent the polarization conductance. Polarization resistance $\left(R_{p}-k \Omega \mathrm{cm}^{2}\right)$ was calculated using relation 1 .

$$
\left(\frac{\mathrm{di}}{\mathrm{dE}}\right)_{\mathrm{E} \rightarrow \mathrm{E}_{\text {corr }}}=\frac{1}{\mathrm{R}_{\mathrm{p}}}
$$

The values of polarization resistance $\left(R_{p}\right)$ increase in glucose, reaching a value of $716 \Omega \mathrm{cm}^{2}$. The numerical values of the electrochemical parameters on behavior of Nitinol in PS and in glucose were calculated using VoltaMaster 4 software with an error of $\pm 1.5 \%$, and are presented in Table 1.

Surface morfology. The electrochemically-corroded Nitinol samples in PS, glucose and aminosteril were also tested using the microscopic images, which indicate the formation of a superficial film providing a passivation on the corroded electrode in these solutions. The microscopic images of Nitinol surface before corrosion (Figure 42a) and after taking place the corrosive processes in PS (Figure 42b) and in glucose (Figure 42c) are presented. 


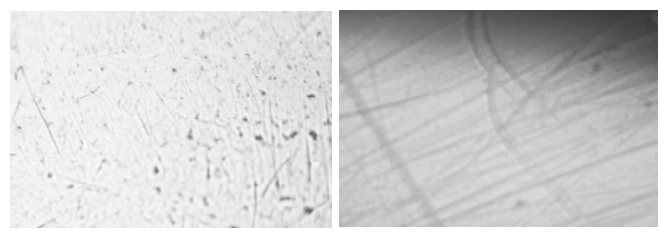

a)

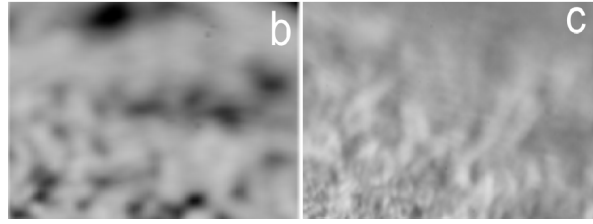

b)

c)

Fig. 42. Microscopic images of the Nitinol surface: a) - before corrosion; b - after corrosion in physiological serum; c - after corrosion in glucose;

Un-corroded sample did not show any particular feature in microscopic imaging than small defects (Figure 6a). The results of microscopy for corroded sample in physiological serum, showed evidence of corrosion pits, and the formation of an un-continuous film on the Nitinol surface was observed. (Figure 42b). In the presence of glucose the micrograph showed no evidence of corrosion pits, but the formation of a more uniform film on the Niti surface was observed (Figure 42c). We can conclude that while correlating the data obtained by the used electrochemical methods it has been observed that the used physiological serum has a quite high corrosive action. It has also been observed that the potential of corrosion in pitting decrease from $504 \mathrm{mV}$ in glucose to $246 \mathrm{mV}$ in physiological serum. All the experimental data obtained show that the physiological serum has a more pronounced corrosive character as compared to a simple solution of $5 \%$ glucose. The results of microscopy for corroded sample in physiological serum, showed evidence of corrosion pits, and the formation of an un-continuous film on the Niti surface was observed. In the presence of glucose the micrograph showed no evidence of corrosion pits, but the formation of a more uniform film on the Nitinol surface was observed.

\subsection{Experiment with NiTi springs used to actuate an external fixator device}

The experiment aims to simulate "in vitro" bone fracture osteosynthesis, which is based on the Nitinol spring actuators and the laws of variation of heating temperature of the Nitinol actuators commuting a mobile bone fragment of a larger fixed bone fragment. This was done by mounting the two Nitinol actuators (like springs) in two cylindrical enclosures equipped with two channels that can slide alonga length determined by the two bolts used to fasten the bone fragments. One of the enclosures is provided with a rectangular cut that serves to mount the temperature sensor.

Materials used:

1. Two Nitinol springs (www.memory-metalle.de)

2. Temperature Sensor TMP 02 (www.sparfun.com)

3. Sharp IR proximity sensor GP2D120XJ00F (www.sparkfun.com)

4. FSR type force sensor (www.interlinkelec.com)

5. PCI data acquisition and control Mega Arduino 2560 (www.arduino.cc)

6. PCI data acquisition and control with Arduino Duemilanove (www.arduino.cc)

7. DFRobot Relay Shield (ww.dfrobot.com)

8. Power supply AC/DC 220/5V, 30 A. 


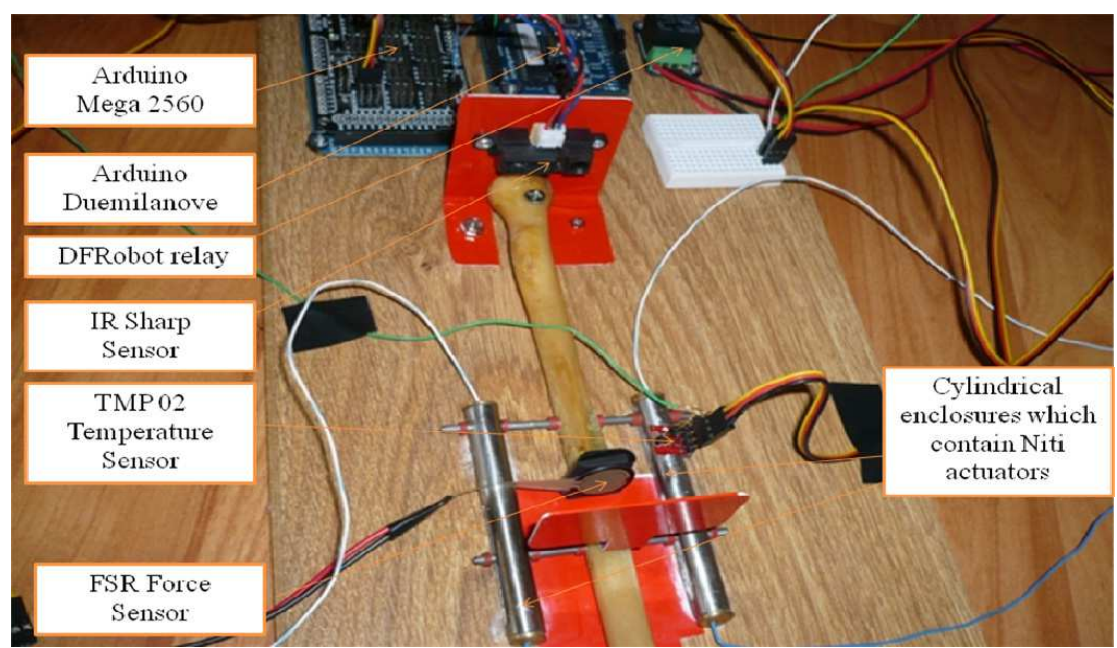

Fig. 43. The experimental layout used to measure the temperature and compression force generated by the Nitinol actuators which can be used in an external fixator device

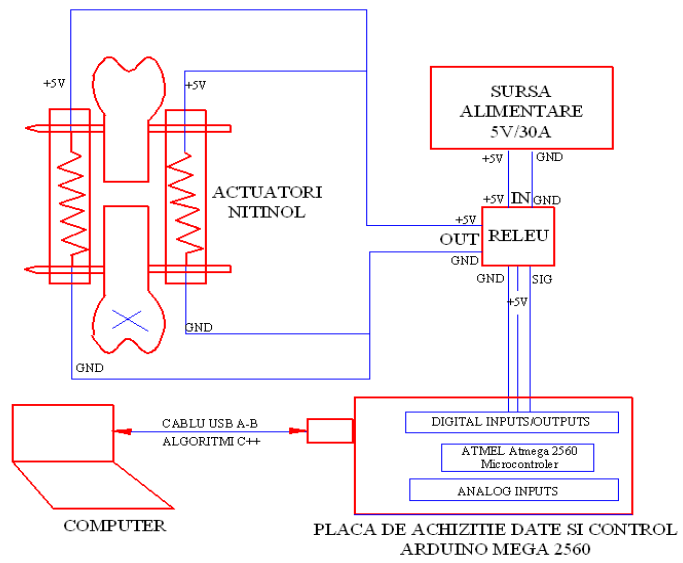

Fig. 44. The actuation scheme used in the experiment

The board data acquisition and control Arduino Mega2560 is designed to collect information from temperature, displacement and force sensors and to return the information on a computer screen, where it can be stored and later processed to obtain graphs and charts characterizing the physical process. The Arduino board is programmed with code written in $\mathrm{C} / \mathrm{C}++$ and communicate with the computer via serial interface. The Arduino Duemilanove board also is loaded with a program, written in $\mathrm{C} / \mathrm{C}++$, which establishes the time interval for opening (actuator of Nitinol are supplied from the power source) or closing (power supply interruption, springs of Nitinol are allowed to cool) the DFRobot relay. The results are shown in Fig. 45 

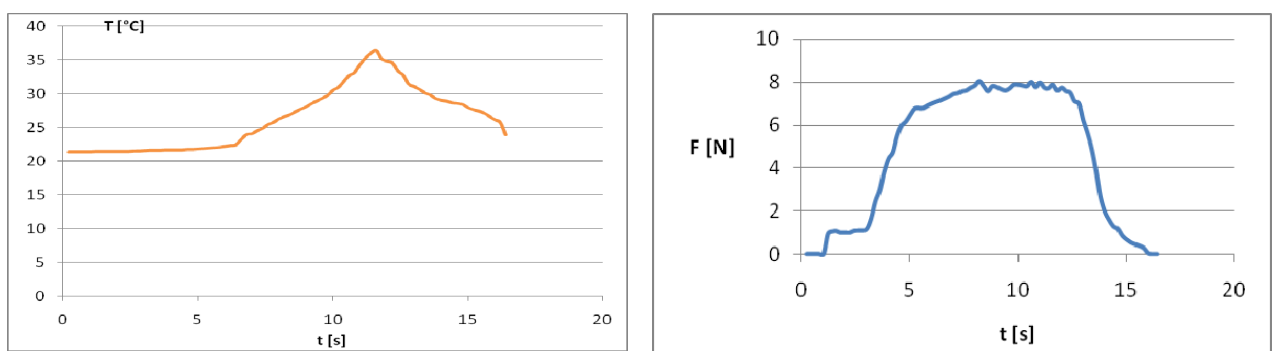

Fig. 45. The evolution of the temperature and bone compression force in time

\subsection{In vitro experiment on the cadaver}

We carried out experiments on the humerus bone of a cadaver.

The surgical technique is the following: One of the module was positioned on the proximal fragment. Using the drilling machine and the spiral, two channels in the cortical were made. Afterwards, two screws were mounted into the channels, making an assembly through which the implant was fixed to the bone. A similar approach involved the distal fragment of the bone. The second implant was positioned on the bone so it could be coupled with the first implant and, at the same time, to allow the longitudinal sliding of the two implants and of the two sectioned bones by $2 \mathrm{~mm}$.

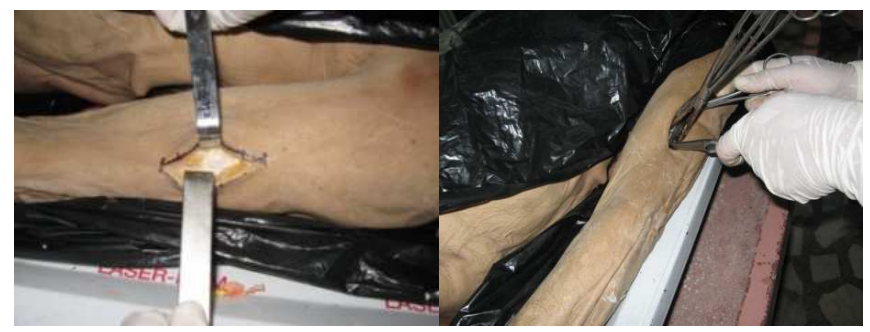

a) b)

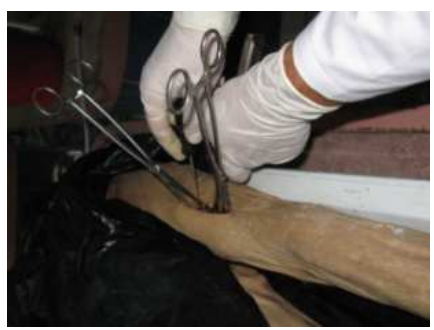

c)

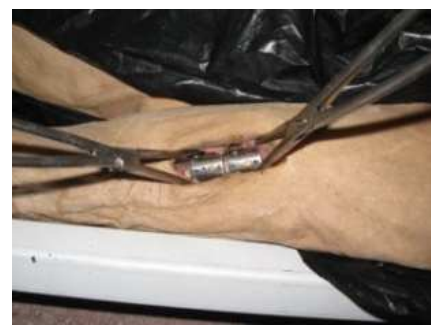

d)

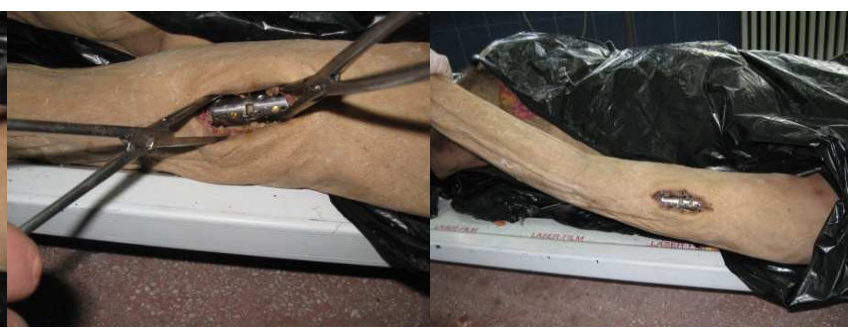

e) f)

Fig. 46. Main stages of the surgical experiment on the humerus bone of a cadaver: a) establishing the fracture zone on the cadaver humerus; $b$ ) the skin is cut; $c$ ) the mounting of the modules; d) the initial coupling of the modules; d) the modules are coupled by two staples; f) the final assembly, before the skin closing. 
After the second implant was mounted by means of the two screws, the implants were coupled by means of the Nitinol staple, which was previously cooled to $-20^{\circ} \mathrm{C}$ in a $\mathrm{NaCl}$ liquid solution kept in a refrigerator. By cooling, the staple is opened and driven into each of the two mounted modules. As the staple warms (possibly just upon heating to body temperature) the pins return to their original shape, pulling the fracture together, determining the translation of the modules and the separated parts of bone are compressed, reducing the risks of wrong orientation or additional bones callus. Main stages of the surgical experiment on the humerus bone of a cadaver are presented in Fig.46.

\section{Acknowledgment}

This work was supported by the funds of Ideas_92 Research Grant

\section{References}

Black, J. (1992). Biological performance of materials:fundamentals of biocompatibility. 2nd edn. New York: Marcel Decker, 1992: 38-60

Cowin, S.C. \& Mehrabadi, M.M.(1987), “On the Identification of Material Symmetry for Anisotropic Elastic Materials", Quarterly Journal of Mechanics and Applied Mathematics, Vol.40, Part 4, pp. 451-476.

Cowin, S.C. \& Mehrabadi, M.M. (1990), ‘Eigentensors of Linear Anisotropic Elastic Materials", Quarterly Journal of Mechanics and Applied Mathematics, Vol.43, pp. 15-41.

Duerig, T.W.; Pelton, A.R. \& Stockel, D. (1999). An overview of Nitinol medical applications, Materials Science and Engineering A, pp. 273-275,149

Farouk, O.; Krettek, C., Miclau, T. et al. (1999). Minimally Invasive Plate Osteosynthesis: Does Percutaneous Plating Disrupt Femoral Blood Supply Less Than the Traditional Technique?, Journal of Orthopaedic Trauma: 1999;13:401-06

Fontana, M.G. (1986). Corrosion engineering: modern theory and applications. 3rd edn. New York: McGraw-Hill, 1986: 445-502

Friend, C.M. \& Morgan, N.B. (1999). Medical applications for Shape-Memory Alloys (SMA), Professional Engineering Publishing Ltd., UK, 1999

Gray's anatomy of the human body, twentieth edition, revised and re-edited by Warren h.Lewis, Newyork: bartleby.com, 2000

Gupta, S. \& Manohar, C.S. (2005) Probability distribution of extremes of von mises stress in randomly vibrating structures, Journal of Vibration and Acoustics Copyright, 2005,Vol.127,547

Heim, U. \& Pfeiffer, K.M. (1988) Internal fixation of small fractures, 3rd Ed. Berlin, SpringerVerlag, 1988:151-160

Hu, K.Z.; Zhang, X.L., Wang, C.T. et al. (2009). The contour of cementless femoral stem has minor effect on initial periprosthetic von Mises stress distribution. A 3-dimensional finite element Saudi Medical Journal, 2009;30:947-951

Jubel, A.; Andermahr, J., Bergmann, H. et al. (2003). Elastic stable intramedullary nailing of midclavicular fractures in athletes, Br J Sports Med 2003;37:480-484

Kregor, P.J.; Stannard, J., Zlowodzki, M. et al. (2001). Distal femoral fracture ixation utilizing the Less Invasive Stabilization System: The technique and early results, 2001,32, Supplement 3, Pages 32-47 
Kapanen, A.; Ilvesaro, J., Danilov, A. et al. (2002). Behaviour of Nitinol in osteoblast-like ROS-17 cell cultures, Biomaterials, 2002;23:645-650

Karahan, O. \& Bingul, Z., Robotics, Automation and Mechatronics, 2008 IEEE Conference, 2008;21-24:78-83

Kul'kova, S.E.; Beketov, K.A., Egorushkin,V.E. \& Muryzhnikova, O.N. (1995), J. Phys. IV 5

Mantovani, D. (2000). Shape memory alloys: Properties and biomedical applications. Journal of the Minerals, Metals and Materials Society, 2000; 52:36-44

Mei, F.; Ren, X. \& Wang, W.T. (1997). The biomechanical effect and clinical application of a Ni-Ti shape memory expansion clamp, Spine 1997, 22, 2083

Muller, M.E.; Nazarian, S., Koch, J. \& Schatzker, J. Fractures classification. AO classification of the long bones fractures, Edition in Rom. language from Tomoaia, Gh., Edit. Risoprint, Cluj Napoca, 2006

Musialek, J.; Filip, P. \& Nieslanik, J. (1998). Titanium-nickel shape memory clamps in small bone surgery, Arch Orthop Trauma Surg 117:341-344.

Patoor, E.; Lagoudas, D., Entchev, P., Brinson, L. \& Gao, X., Shape memory alloys, Part I: General properties and modeling of single crystals, Mechanics of Materials, 38 (2006) 391-429

Pelton, A.R.; Stochel, D. \& Duerig, T.W. (2000). Medical uses of Nitinol, Mater Sci Forum, 2000:327-328:63-70.

Psalman, V. (2008). Facta Universitatis, Series: Physical Education Sport, Dynamic balance and its diagnostics by using 3D biomechanical analysis. 2008;6:105-109

Raghubir, S. \& Dahotre, B., Corrosion degradation and prevention by surface modification of biometallic materials, Journal of Materials Science: Materials in Medicine, Volume 18(5):725-751, DOI: 10.1007/s10856-006-0016-y

Rychlewski, J. \& Zhang, J.(1989), Anisotropy degree of elastic materials. Arch. Mech. 47 (5), 697-715.

Ryhänen, J.; Niemi, E., Serlo, W. et al. (1997). Biocompatibility of nickel-titanium shape memory metal and its corrosion behaviour in human cell cultures. J BIOMED MATERRES 1997; 35: 451-7.

Ryhanen, M.; Kallionen, J. \& Tuukkanen J. (1999). Medical applications for Shape-Memory Alloys (SMA), Professional Engineering Publishing Ltd., 1999:53

Samide, A.; Bibicu, I., Oprea, B. \& Tutunaru, B. (2009) J.Optoelectronics Advanced Materials, 102156 (2008)

Sanders, J.O.; Sanders, A.E., More, R. et al. Electrochemical and surface characterization of a nickel-titanium alloy, Spine, 1993,18,1640.

Shabalovskaya, S.A. Biological aspects of TiNi alloy surfaces, Journal de Physique IV, C8, 5, 1995, 5/2(8):C8.1199-C8.12041199

Shabalovskaya, S.A. On the nature of the biocompatibility and on medical applications of NiTi shape memory and superelastic alloys. J BioMed Mater Res 1996;6: 267-89

Shrier, L.L.; Jarman, R.A. \& Burstein, O.T. Corrosion-metal/environment reactions. 3rd edn. Jordan Hill, Oxford: Butterworth-Heinemann, 1995: 2: 3-2: 164

Smith, G.F. \& Rivlin, R.S., "The Strain Energy Function for Anisotropic Elastic Materials", Transactions of the American Mathematical Society, Vol.88, pp.175-193, 1958.

Sutcliffe, S. (1992), "Spectral Decomposition of the Elasticity Tensor ", Journal of Applied Mechanics, Vol. 59, pp 761-773. 
Tarnita, D.; Tarnita, D.N., Tarnita, R., Berceanu, C. \& Cismaru, F. (2010). Modular adaptive bone plate connected by Nitinol staple, Materialwissenschaft und Werkstofftechnik, Materials Science and Engineering Technology, Special Edition Biomaterials, Willey-Vch., Matwer 40 (1-2) 1-120 (2009) ISSN 0933-5137

Tarnita, D.; Bolcu, D., Berceanu, C. \& Cismaru, F. (2010).Theoretical and experimental studies for an orthopedic staple made up Nitinol, Journal of Optoelectronics and Advanced Materials, Vol. 12, No. 11, November 2010, p. 2323 - 2332 (0,33626)

Theocaris P.S. \& Philippidis, E.(1989), "Elastic Eigenstates of a medium with transverse Thoma, T.Y., Proc Nat. Acad. Sci. U S A. 1955 November 15; 41(11), 908

Ting, T.C.T. (1987), "Invariants of Anisotropic Elastic Constants", Quarterly Journal of Mechanics and Applied Mathematics, Vol.40, Part 3, pp. 431-448.

Trepanier, C.; Tabrizian, M., Yahia, L.H. et al. Effect of the modification of the oxide layer on NiTi stent corrosion resistance, JBMR (Appl Biomater) 1998; 43: 433-40

Tsung-Jung, H., Wei-Hsiang, L., Journal of the Chinese Institute of Engineers, 2010, 33, No.1,121

von Mises, R., Mechanik der Festen Korper im plastisch deformablen Zustand. Göttin. Nachr. Math. Phys., 1913;1:582-592

Venugopalan, R. \& Trépanier, C. Assessing the corrosion behaviour of Nitinol for minimally-invasive device design, Min Invas Ther \& Allied Technol 2000: 9(2) 6774

Wever, D.J.; Veldhuizen, A.G., de Vries, J. et al. Electrochemical and surface characterization of a nickel-titanium alloy. Biomaterials 1998; 19: 761-9

Yang, P.J.; Zhang, Y.F., Ge, M.Z. et al. Internal fixation with Ni-Ti shape memory alloy compressive staples in orthopaedic surgery: a review of 51 cases. Chin Med J (Engl) $1987,100,712$

Yeung, K.W.; Poon, R.W., Chu, P.K. et al. Cheung Journal of Biomedical Materials Research Part A. Aug 2007, Vol. 82A, No. 2: 403-414 


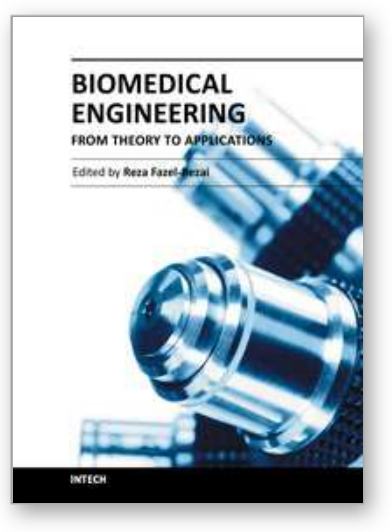

\author{
Biomedical Engineering - From Theory to Applications \\ Edited by Prof. Reza Fazel
}

ISBN 978-953-307-637-9

Hard cover, 486 pages

Publisher InTech

Published online 29, August, 2011

Published in print edition August, 2011

In all different areas in biomedical engineering, the ultimate objectives in research and education are to improve the quality life, reduce the impact of disease on the everyday life of individuals, and provide an appropriate infrastructure to promote and enhance the interaction of biomedical engineering researchers. This book is prepared in two volumes to introduce a recent advances in different areas of biomedical engineering such as biomaterials, cellular engineering, biomedical devices, nanotechnology, and biomechanics. It is hoped that both of the volumes will bring more awareness about the biomedical engineering field and help in completing or establishing new research areas in biomedical engineering.

\title{
How to reference
}

In order to correctly reference this scholarly work, feel free to copy and paste the following:

Daniela Tarnita, Danut Tarnita and Dumitru Bolcu (2011). Orthopaedic Modular Implants Based on Shape Memory Alloys, Biomedical Engineering - From Theory to Applications, Prof. Reza Fazel (Ed.), ISBN: 978-953307-637-9, InTech, Available from: http://www.intechopen.com/books/biomedical-engineering-from-theory-toapplications/orthopaedic-modular-implants-based-on-shape-memory-alloys

\section{INTECH}

open science | open minds

\section{InTech Europe}

University Campus STeP Ri Slavka Krautzeka 83/A 51000 Rijeka, Croatia Phone: +385 (51) 770447

Fax: +385 (51) 686166 www.intechopen.com

\section{InTech China}

Unit 405, Office Block, Hotel Equatorial Shanghai No.65, Yan An Road (West), Shanghai, 200040, China 中国上海市延安西路65号上海国际贵都大饭店办公楼405单元 Phone: +86-21-62489820

Fax: +86-21-62489821 
(C) 2011 The Author(s). Licensee IntechOpen. This chapter is distributed under the terms of the Creative Commons Attribution-NonCommercialShareAlike-3.0 License, which permits use, distribution and reproduction for non-commercial purposes, provided the original is properly cited and derivative works building on this content are distributed under the same license. 